Revue des patrimoines

\title{
Architectures et nations. Le cas du Schleswig au regard d'autres régions frontalières en Europe
}

Architecture and regions, the case of Schleswig compared to other border

regions in Europe

\section{Peter Dragsbo}

\section{OpenEdition Journals}

Édition électronique

URL : http://journals.openedition.org/insitu/21066

DOI : $10.4000 /$ insitu. 21066

ISSN : 1630-7305

Éditeur

Ministère de la culture

Référence électronique

Peter Dragsbo, «Architectures et nations. Le cas du Schleswig au regard d'autres régions frontalières en Europe », In Situ [En ligne], 38 | 2019, mis en ligne le 06 mars 2019, consulté le 30 avril 2019. URL : http://journals.openedition.org/insitu/21066 ; DOI : 10.4000/insitu.21066

Ce document a été généré automatiquement le 30 avril 2019

\section{c) $(9)$}

In Situ Revues des patrimoines est mis à disposition selon les termes de la licence Creative Commons Attribution - Pas d'Utilisation Commerciale - Pas de Modification 4.0 International. 


\title{
Architectures et nations. Le cas du Schleswig au regard d'autres régions frontalières en Europe
}

\author{
Architecture and regions, the case of Schleswig compared to other border \\ regions in Europe
}

Peter Dragsbo

Smith.

1 Le présent article est le fruit d'un projet entrepris de 2013 à 2014 et qui relève des initiatives de recherche lancées par le Museum Sønderjylland - Sønderborg Slot (musée du Jutland du Sud - château de Sønderborg), musée consacré à l'histoire danoise et allemande de la région frontalière du Schleswig/Slesvig. Dans la continuité d'enquêtes déjà anciennes portant sur les échanges et brassages culturels dans cette région à la frontière entre le Danemark et l'Allemagne, j'ai voulu élargir les perspectives pour faire une comparaison de l'architecture d'un certain nombre d'autres régions frontalières qui ont connu, au cours de leur histoire, des changements d'appartenance nationale, d'identité et de gouvernement. Mon regard s'est tourné en premier lieu vers les anciennes régions frontalières allemandes ayant connu des changements de nationalité au lendemain de la Première Guerre mondiale : l'Alsace (Elsaß), la Lorraine (Lothringen) et, à l'ouest de la Pologne, les anciennes provinces de Posen (Poznań) et Westpreussen (PrusseOccidentale). Pour prendre en compte des situations plus contrastées, j'ai choisi d'examiner également le Tyrol du Sud (ou Haut-Adige) (Südtirol), une région à la frontière entre l'Italie et l'Autriche, annexée par l'Italie après 1918. D'autres territoires frontaliers, comme la région germanophone d'Eupen-Malmédy en Belgique, la région à la frontière entre la Finlande et la Russie ou encore certaines régions hongroises ont été considérées mais ont été écartées faute de temps pour les étudier et de littérature accessible ${ }^{1}$.

2 La période couverte par notre étude s'ouvre dans les années 1840-1850, époque à laquelle, dans le contexte de renouveau des styles « historiques » en Europe, émergent les débats 
sur les liens entre architecture et nationalité. Elle prend fin avec la Seconde Guerre mondiale, qui clôt les discours nationalistes et traditionalistes en architecture, à l'exception de l'architecture stalinienne de l'Europe de l'Est. Notre recherche s'appuie sur de multiples précédents, en particulier sur l'étude comparative du romanticisme national et de l'architecture moderne en Allemagne et dans les pays scandinaves publiée par l'historienne de l'art américaine Barbara Miller Lane ${ }^{2}$. Autres sources utiles, les travaux du Dr Niels Wilcken, de Sarrebruck, qui traitent des administrations nationales et municipales des bâtiments pendant la période 1871-1918, lorsque l'Alsace-Lorraine était allemande ${ }^{3}$; le projet GrenzWerte (valeurs des frontières), une recherche collaborative entre le Danemark et l'Allemagne sur l'architecture de part et d'autre de la frontière entre 1912 et 2012, projet dans lequel le musée du Jutland du Sud - château de Sønderborg a joué un rôle majeur ${ }^{4}$; dernier modèle pour nous, mais non des moindres, le projet de coopération architecturale franco-allemande intitulé «Interférences/ Interferenzen ", un projet associant les Villes de Strasbourg et de Francfort-sur-le-Main entrepris de 2009 à $2014^{5}$. N'oublions pas, enfin, les importantes recherches de Christiane Pignon-Feller sur l'histoire de l'urbanisme et de l'architecture à Metz à l'époque wilhelmienne (Deuxième Reich) ${ }^{6}$. Au cours de mes propres recherches sur le terrain, j'ai également bénéficié d'échanges très riches et très utiles, en particulier avec Marie Pottecher, conservatrice du patrimoine à Strasbourg et d'autres membres des équipes strasbourgeoises, ainsi qu'avec le Dr Wittfrida Mitterer à Bozen/Bolzano, le DrJan Skuratowicz et le professeur Dr Tadeusz Zuchowski, de l'université Adam Mickiewicz de Poznań, et le Dr Christoph Hölz, de l'Archiv für Baukunst à Innsbruck.

\section{Nations et architecture}

3 L'un des premiers enjeux de notre recherche concernait les liens possibles entre la fierté d'une nation, son amour-propre, et les caractéristiques stylistiques de son architecture. Au préalable, il fallait aborder la question des relatio ns entre «État » et « nation » et la différence entre les fondements «culturels » et "politiques » d'une nation. Autrement dit, il fallait faire la distinction entre le principe occidental de l'État nation et le principe oriental de nation en tant que culture. Il est intéressant de noter que les nations «culturelles» ont eu tendance à se légitimer à travers des symboles sélectionnés, réinterprétés ou empruntés qui cherchent à établir le fait que la nation en question a des racines profondes ou peut-être récentes mais interprétées comme des racines anciennes.

C'est pour cette raison que notre comparaison entre les différentes régions frontalières analysées est centrée sur la place de l'architecture et du style dans les «conflits entre nations ", y compris les irredenta, c'est-à-dire les mouvements irrédentistes, luttant pour l'autonomie, pour l'indépendance ou pour l'affiliation à une mère-patrie nationale. Il s'avère en effet que certains mouvements sécessionnistes ont développé leur propre " contre-architecture ", surtout lorsqu'ils se trouvaient sous la domination de nations aux langages architecturaux particulièrement forts. Alternativement, un conflit national a pu également aboutir à un consensus architectonique, fondé sur une identité régionale ${ }^{7}$.

L'architecture européenne des $\mathrm{XIX}^{\mathrm{e}}$ et $\mathrm{xx}^{\mathrm{e}}$ siècles a été caractérisée par des interactions continues entre des thèmes nationaux et universels. Au cours de la première moitié du $\mathrm{XIX}^{\mathrm{e}}$ siècle, l'historicisme, qui comprenait le style comme le produit d'une époque et d'un lieu, a succédé au classicisme avec ses notions de valeurs éternelles, et a occasionné la recherche de styles nationaux. À partir de 1860, le discours de l'architecture nationale est 
remplacé par un historicisme commun à l'Europe. Les nations rivalisent entre elles en cherchant à se montrer comme les championnes de la civilisation, symbolisée par les grands styles tels que le gothique français ou la Renaissance italienne ${ }^{8}$. Parmi ces nations en compétition, on trouve la Prusse, la France, la Russie, l'Autriche-Hongrie et les pays scandinaves. Au cours des années 1890 cependant, une tendance de retour aux thèmes nationaux émerge à travers le développement du romanticisme national, qui se focalise dorénavant sur de vrais conflits et contradictions nationaux, et englobe des éléments empruntés au patrimoine national, à la culture folklorique et à l'architecture vernaculaire. Ainsi, vis-à-vis des pays voisins mais aussi de leurs propres minorités, les nations manifestent leur fierté dans des monuments et des musées nationaux et surtout, dans les Expositions universelles 9

(fig. 1)

Figure 1

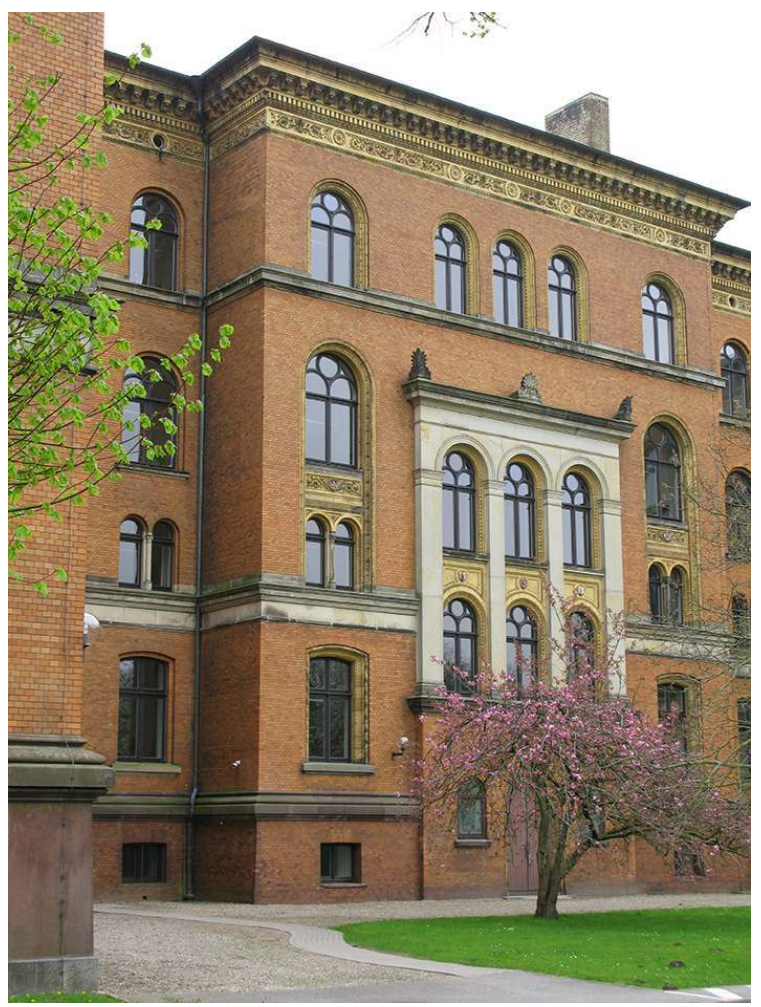

Le bâtiment administratif de la chambre provinciale de Schleswig-Holstein dans la ville de Schleswig Connu comme "L'éléphant rouge », ce bâtiment a été construit en brique en 1875 par le professeur Heinrich Köhler (1830-1903), Hanovre, dans un style conventionnel Renaissance italienne, montrant la Prusse comme porteuse de valeurs universelles.

Phot. Peter Dragsbo. (c) Peter Dragsbo.

7 Suite à la vague des mouvements de réforme en Europe autour de 1900, une opposition aux « grands styles » s'est fait jour, recherchant des traditions régionales et vernaculaires intimement associées, dans des pays multiculturels come l'Allemagne ou la Suisse, avec le concept de Heimat ${ }^{10}$. Aux lendemains de la Première Guerre mondiale, de nombreux pays d'Europe, en particulier les grands perdants du conflit, comme l'Allemagne et l'Autriche, ont connu une rupture radicale avec leur passé, suivie d'un développement de l'architecture expressionniste et moderniste. Les nouveaux États ou les États nouvellement recréés cherchaient à exprimer à la fois leur place dans l'Europe moderne 
et leurs spécificités nationales. Ils hésitaient entre le modernisme et le néo-classicisme d'un côté, comme symboles d'une appartenance européenne, et le romanticisme national de l'autre, en opposition aux grands styles des anciennes hégémonies, l'Autriche-Hongrie, la Russie ou l'Empire ottoman ${ }^{11}$.

\section{Schleswig/Slesvig, conflits nationaux et changements de style}

8 La région du Schleswig (Slesvig), située à la frontière entre le Danemark et l'Allemagne, est jusqu'à la fin du Moyen Âge un territoire de langue danoise, mais qui au XIII ${ }^{e}$ siècle, fut séparée du royaume danois pour devenir un duché indépendant. À partir de 1460 , le Schleswig fut réuni à la province germanophone du Holstein, mais sous la tutelle personnelle du roi danois. Le Holstein conserva alors son statut d'État-membre de l'Empire germanique. Au cours des siècles suivants, le Schleswig subit de plus en plus l'influence de la langue et de la culture allemandes, devenant ainsi une région que l'on qualifierait aujourd'hui de multi-ethnique et multiculturelle au sein de l'État danois, le Gesamtstaat (1773-1864). Au cours de la première moitié du XIX ${ }^{\mathrm{e}}$ siècle, deux mouvements nationaux-libéraux se développèrent en parallèle dans le Schleswig-Holstein, l'un danois et l'autre allemand, tous deux revendiquant des droits sur l'ensemble du territoire. Ce conflit dégénéra en guerre civile, la première guerre du Schleswig, de 1848 à 1850 . En dépit de la victoire danoise, le problème de l'appartenance du Schleswig demeura sans solution. Lorsque le Danemark rompit les accords internationaux signés à la fin de la première guerre, la deuxième guerre du Schleswig éclata, en 1864. Cette fois-ci, la Prusse et l'Autriche intervinrent en faveur de l'union allemande. Après la défaite du Danemark, les duchés furent placés sous une administration commune de la Prusse et de l'Autriche mais à la suite de la guerre austro-prussienne de 1866, ils furent annexés par la seule Prusse pour devenir, entre 1871 et 1920, une province du Deuxième Reich. Après la Première Guerre mondiale, un référendum fut organisé, donnant lieu à la partition du territoire et à l'établissement d'une frontière qui suit, sensiblement, la division entre les majorités allemande et danoise. C'est l'une des rares frontières de cette époque, fixée par référendum, qui subsiste encore aujourd'hui ${ }^{12}$.

Comme d'autres pays européens du milieu du xix siècle, le Danemark a cherché un style architectural national. Parmi d'autres possibilités, on identifia ce style dans la Renaissance hollandaise de l'époque du célèbre roi Christian IV, au début du XVII ${ }^{\mathrm{e}}$ siècle. Ce style se développa particulièrement après l'incendie du château royal de Frederiksborg, en 1859, et dans les réflexions qui entourèrent sa reconstruction ${ }^{13}$. On observe une recherche similaire d'un style national dans le duché de Schleswig-Holstein, où la noblesse, plutôt allemande, cultiva le style néogothique comme symbole d'indépendance à la fois du classicisme officiel danois et de l'« hellénisme» prussien. Après l'annexion par la Prusse, en 1866, les autorités de l'architecture officielle passèrent sous l'influence de l'idéologie architecturale prussienne, qui cherchait alors à positionner la Prusse comme l'une des grandes puissances d'Europe. Ainsi, des bâtiments-symboles de la nouvelle domination prussienne, casernes ou bâtiments administratifs, ont-ils été conçus dans différentes versions de la Renaissance italienne, tandis que l'élite locale du Schleswig-Holstein continuait à préférer le style néogothique de l'« École de Hanovre » élaboré par des architectes tels que Conrad Wilhelm Hase (1818-1902) et Johannes Otzen $(1839-1911)^{14}$. 
Figure 2

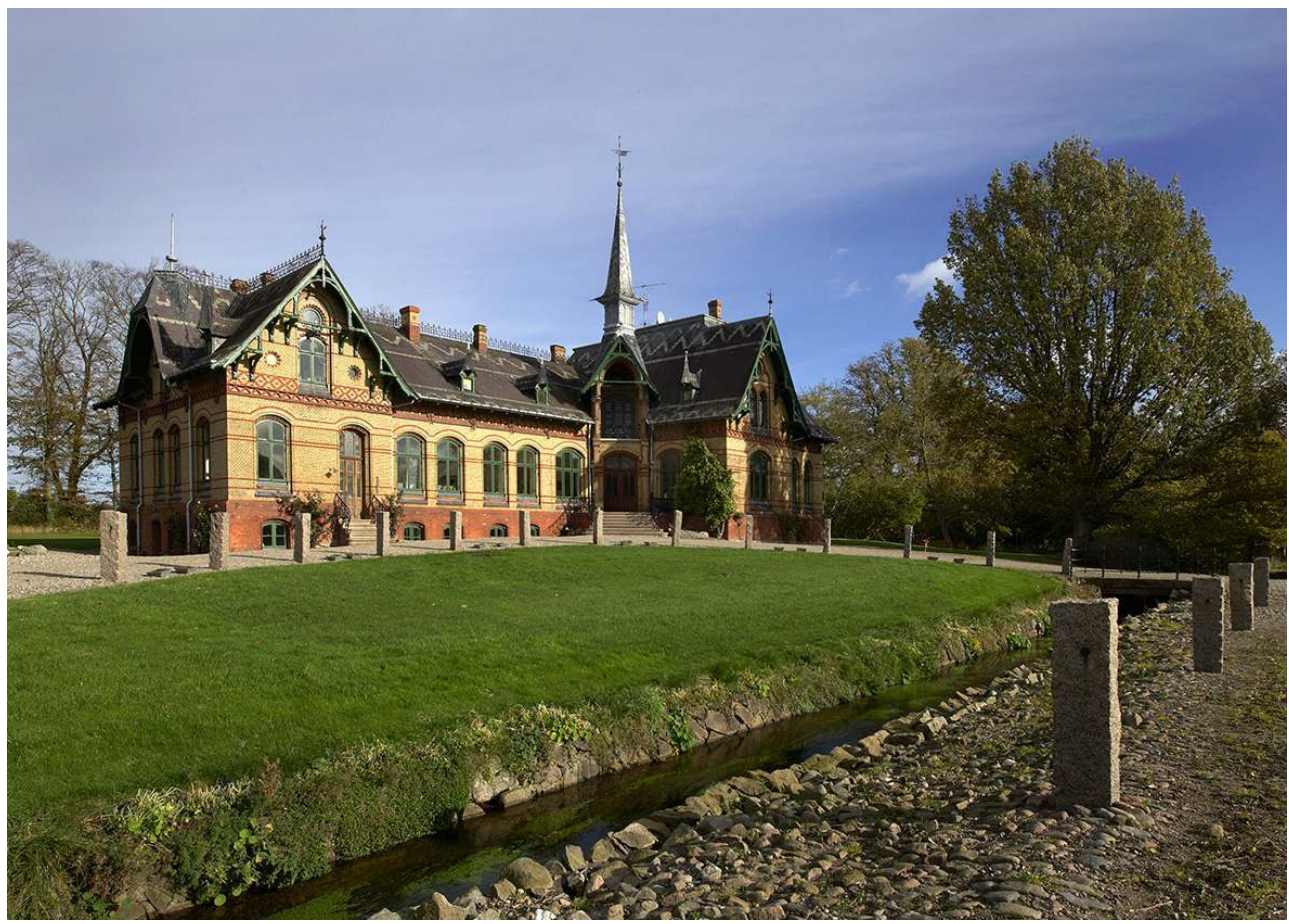

Le manoir d'Ultang près de Haderslev, conçu en 1884 par l'architecte A. W. Prale, originaire de Flensburg. C'est un exemple unique du style néogothique de l'école de Hanovre.

PHOT. SøREN PETERSEN. ( ) SøREN PETERSEN.

11 Au cours des premières années du Deuxième Reich, les autorités voulurent éviter toute forme de séparatisme régional ou religieux. Vers 1890 toutefois, la puissance impériale se sentit plus assurée et commença à tolérer l'émergence d'identités régionales qui, avec le concept de Heimat, cherchaient à contrôler les immenses différences culturelles et historiques au sein du Reich. Un de ces courants régionalistes est né des travaux de Julius Langbehn (1851-1907). Dans ses écrits, cet auteur prétendait que les Allemands du Nord, avec les populations des Pays-Bas et les Danois, étaient les « vrais » Allemands. Ses idées purent prendre racine dans un sol accueillant et fertile et contribuèrent à l'émergence d'un romanticisme national particulier dans l'Allemagne du Nord ${ }^{15}$. Ainsi, à partir de 1890, le romantisme national, appelé märkisches Gotik, fut-il le style dominant dans les bâtiments officiels des provinces prussiennes. C'est un style relativement libre, en brique, influencé par les bâtiments de l'ancienne Ligue hanséatique et par les églises médiévales, les châteaux et les portes monumentales des villes de la Marche de Brandebourg. Ce style s'imposa dans la majorité des bâtiments prussiens officiels jusqu'aux années qui précédèrent la Première Guerre mondiale ${ }^{16}$. Dans la partie nord du Schleswig, le Baurat (inspecteur du service d'urbanisme) Friedrich Wilhelm Jablonowski (1854-1932) eut la responsabilité de l'architecture officielle, imprimant ainsi sa marque sur cette région frontalière.

(fig. 3) 
Figure 3

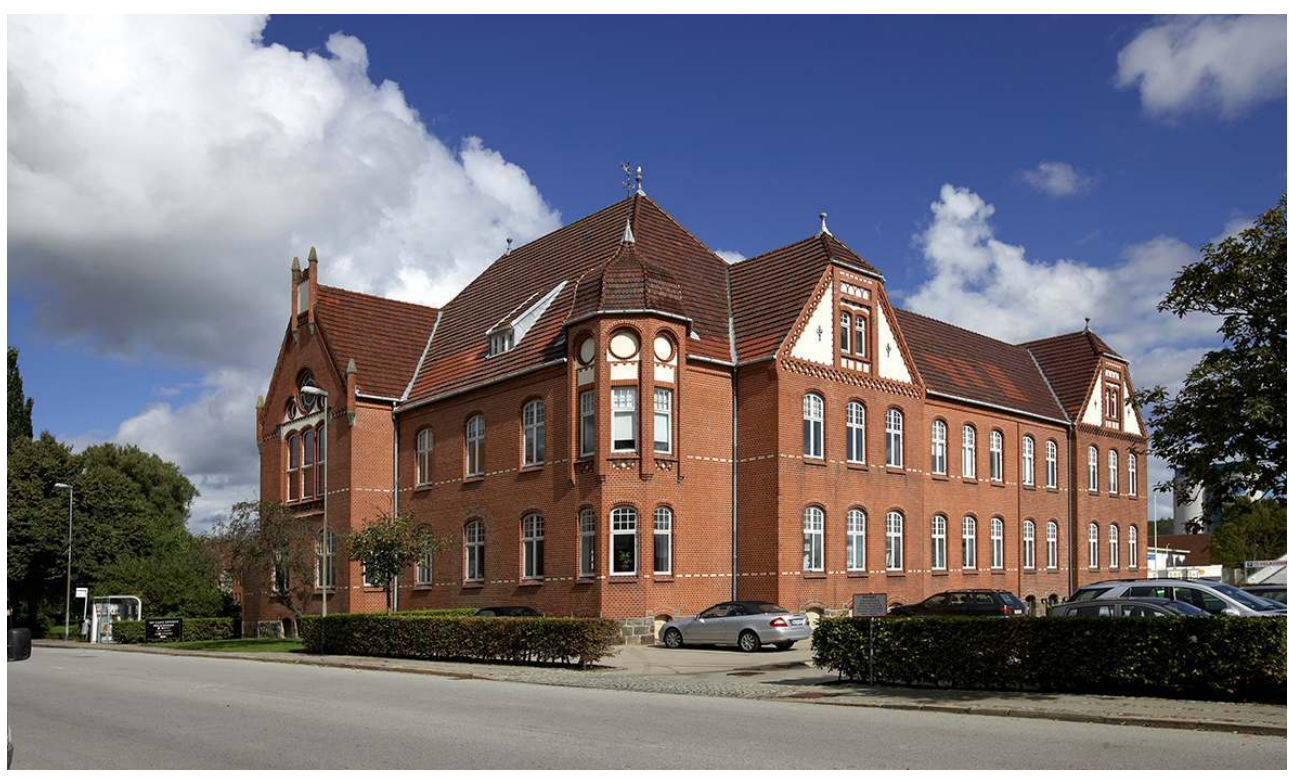

Ancien bâtiment de l'hôtel de l'administration locale à Aabenraa, construit de 1902 à 1904. C'est un bon exemple du style gothique « märkisch » officiel. Le bâtiment a été conçu par l'architecte du comté, Friedrich Wilhelm Jablonowski.

PHOT. SøREN PETERSEN. @ S SøREN PETERSEN.

Dans le Schleswig du Nord, le projet national le plus important au cours de ces années fut la construction de deux grands ensembles formant le quartier général de la marine, à Flensburg et à Sønderborg. Ce projet relevait du renforcement de la marine allemande de haute-mer et de son transfert partiel depuis son ancienne base de Kiel. Bien que le premier projet pour ce quartier général ait été conçu par l'architecte préféré de l'empereur Guillaume II, Franz Schwechten (1841-1924), ce sont les architectes, Adalbert Kelm (1856-1939) à Flensburg et Eugen Fink (1878-1955) à Sønderborg qui en signèrent finalement les plans. L'architecture d'Adalbert Kelm est assez conventionnelle, ses motifs s'inspirant des mairies et des châteaux des villes hanséatiques. À Sønderborg, les bâtiments d'Eugen Fink sont d'un style plus libre, prenant leur inspiration du château médiéval de Marienburg (Malbork) en Prusse-Orientale, avec quelques éléments d'Art nouveau, le Jugendstii ${ }^{17}$.

(fig. 4) 


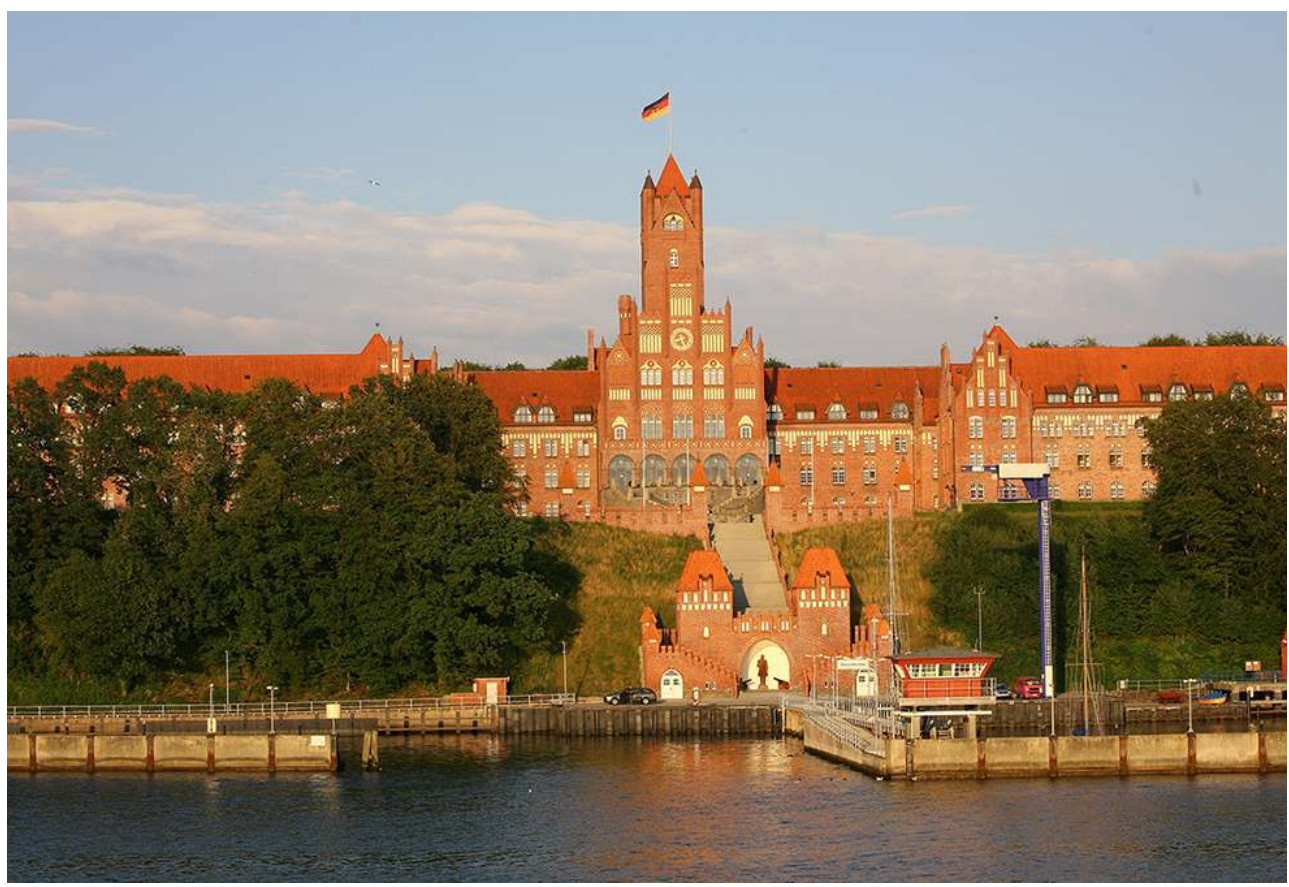

Le quartier-général de la marine à Mürwik, près de Flensburg, conçu par l'architecte naval Adalbert Kelm. Réalisé dans un style gothique hanséatique, il se voulait un " Marienburg à côté du fjord de Flensburg ». La marine pouvait ainsi se présenter à la fois comme l'héritière d'un ordre allemand du Moyen Âge et comme une institution moderne du Reich.

PHOT. DENKMALAMT FLENSBURg. (C) DENKMALAMT FLENSBURg.

Pendant ce temps, l'empereur Guillaume II, en collaboration avec Franz Schwechten, recherchait une architecture nationale qui soit appropriée à l'Empire allemand unifié. C'est ainsi que se développa un romanticisme national inspiré des monuments romans du XII ${ }^{\mathrm{e}}$ siècle, le palais impérial (Kaiserpfalz) de Goslar (Basse-Saxe), par exemple, ou encore le château de Dankwarderode à Brunswick (Basse-Saxe). S'établit ainsi une revendication symbolique de continuité entre la dynastie médiévale des Hohenstaufen et la dynastie plus récente à laquelle appartenait Guillaume II, les Hohenzollern de Brandebourg ${ }^{18}$. Ce style impérial s'est manifesté d'abord dans la construction, à Berlin, à partir de 1890, de l'église du Souvenir-de-l'empereur-Guillaume (Kaiser-Wilhelm-Gedächtniskirche), mais il s'est déployé par la suite dans les régions frontalières contestées et en particulier dans les villes désignées comme "forteresses frontalières ", telles Metz en Lorraine et Poznań (Posen) en Prusse-Occidentale. Pour la construction du quartier général de la marine dans le Schleswig, ce style impérial ne fut pas retenu. La marine allemande se considérait à la fois comme l'héritière de l'ordre teutonique médiéval et comme une administration moderne au sein de l'Empire, indépendante donc des lubies du Kaiser ${ }^{19}$. En raison de la répression des sympathies danoises dans les populations du Schleswig du Nord, le romanticisme national prussien était vu par ces populations comme le symbole même de la domination allemande. En 1908, un représentant important du mouvement danois put écrire que «ces bâtiments seraient hideux, peu importe leur localisation, mais dans le Schleswig du Nord, ils sont doublement hideux $»^{20}$.

À la même époque, un mouvement de réforme sociale et culturelle se développait partout en Europe, y compris dans l'Empire allemand et au Danemark. En termes 
architectoniques, ce mouvement exprimait une réaction à l'égard des "grands styles » de l'historicisme et du romanticisme national. En Allemagne, de nouvelles lois d'urbanisme comprenaient des règlements pour des zones de "construction rurale", et les idées anglaises autour des cités-jardins purent se développer grâce au financement, en 1902, du Deutsche Gartenstadt-Gesellschaft, l'association allemande des cités-jardins. Le mouvement Heimatschutz, au cours des années 1890, et les livres Kulturarbeiten, écrits par Paul Schultze-Naumburg (1869-1949) et publiés par le Kunstwart de 1901 à 1917 exercèrent une influence particulière. En 1904, l'union allemande des Heimatschutz fut fondée, suivie par le lancement d'un grand nombre de sociétés de Heimatschutz et de Baupflege (protection du patrimoine) $)^{21}$.

(fig. 5)

Figure 5

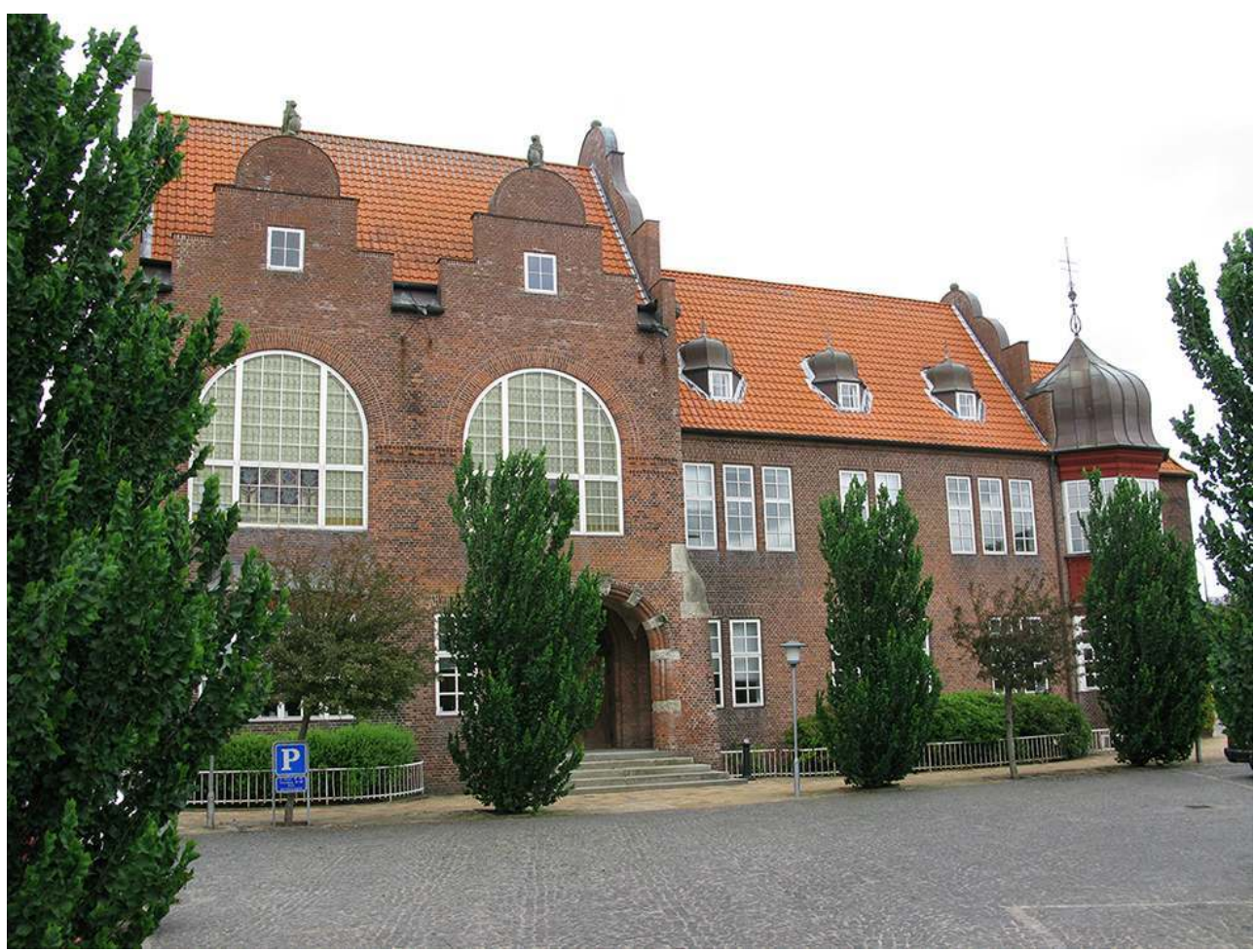

L'hôtel de l'administration locale à Tønder, construit en 1907 par les architectes berlinois Paulus, Dinklage et Lilloe, représente l'une des premières tentatives dans le Schleswig de construire suivant une tradition régionale. Mais la tradition est plus celle de la Frise que celle du Schleswig.

PHOT. SøREN PETERSEN. @ SøREN PETERSEN.

Cette vague de réformes architecturales arriva dans le Schleswig du Nord avec la construction, en 1908, de bâtiments de l'administration provinciale à Tønder (Tondern), conçus, après concours public, par les architectes Ernst Paulus (1868-1935), August Dinklage (1849-1920) et Olaf Lilloe (1872-1943). Plus tard, le maire allemand de cette province, Friedrich Wilhelm Rogge, en collaboration avec les architectes locaux membres du Heimatschutz, lança l'association Baupflege Tondern, la première de son genre dans le Schleswig-Holstein. En seulement quelques années, par la publicité, la formation des maitres d'œuvre et la documentation autour des traditions vernaculaires, cette association réussit à éveiller une nouvelle appréciation du "bâti domestique ", appréciation partagée par les instances officiellement chargées des permis de construire 
et les bâtisseurs privés. Les vieilles maisons de Møgeltønder (Mögeltondern) en particulier, un village des marais, servirent de modèles pour ce nouveau style vernaculaire, devenu rapidement le Heimatstil du Schleswig-Holstein ${ }^{22}$. L'un des premiers édifices de ce style fut le lycée allemand de Tinglev (Tingleff), construit en 1908 par Paulus, Dinklage et Lilloe, un établissement conçu pour répondre aux programmes éducatifs du mouvement danois.

Figure 6

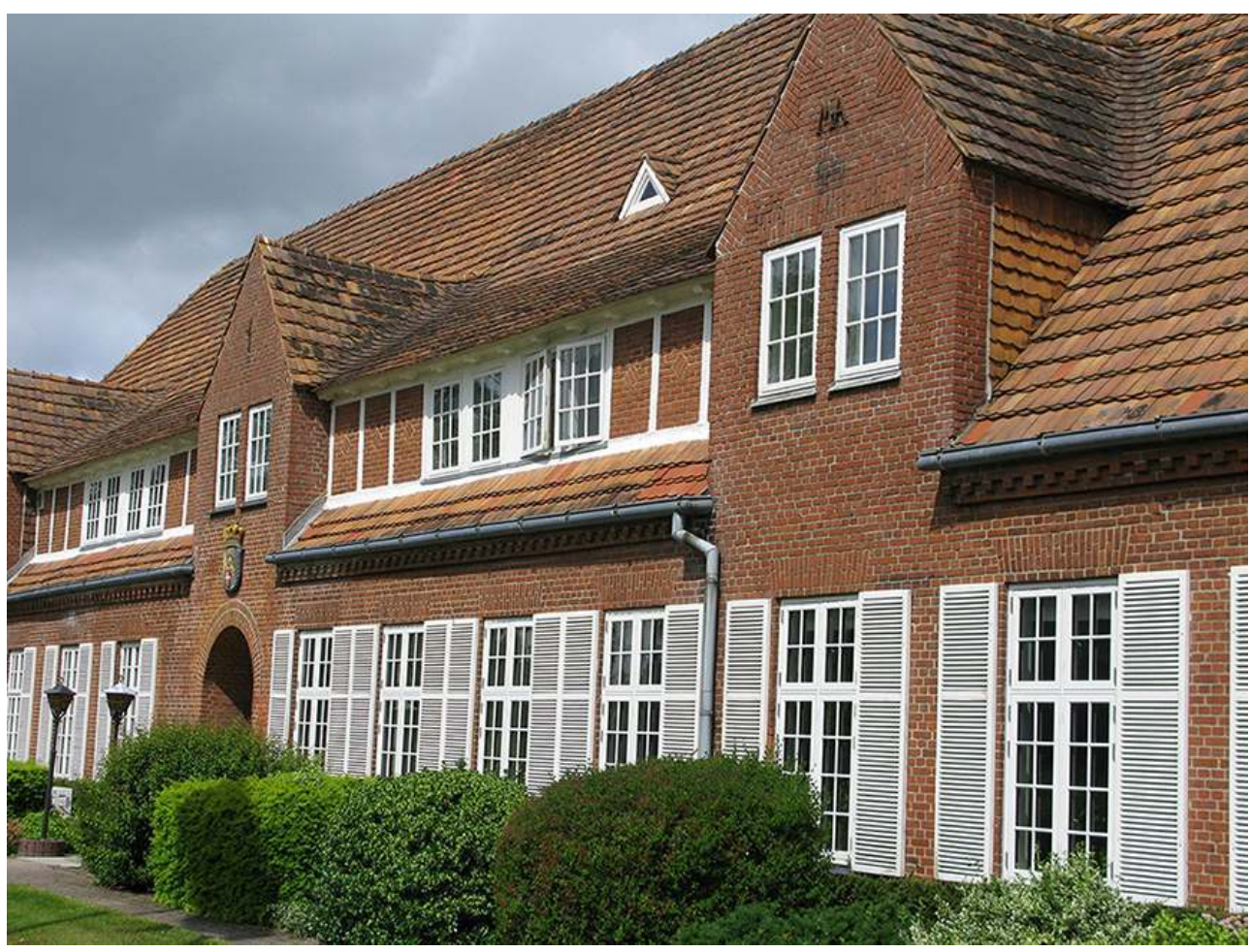

Une autre œuvre des architectes Paulus, Dinklage et Lilloe, le lycée allemand de Tinglev, de 1908. Avec ses frontons de Frise et ses volets blancs, l'édifice est davantage en accord avec les anciennes traditions du Møgeltønder. Seules les tuiles en forme de queue de castor viennent du Heimatstil de Saxe.

PHOT. SøREN PETERSEN. @ S SøREN PETERSEN.

20 Au début $d u x^{e}$ siècle, une nouvelle contre-architecture émerge sous l'influence du mouvement danois du Schleswig du Nord. Au cours des années 1890, ce mouvement danois réussit à créer une société parallèle au sein de la société, inspirée par le dynamisme de la vie démocratique de l'« ancien » Danemark. Un besoin se fait sentir pour de nouvelles salles communales et de nouvelles églises libres où la population danoise pourrait se réunir, indépendamment des autorités allemandes qui harcèlent la vie publique des Danois. À l'origine, ces salles communales n'étaient que des maisons ordinaires en brique avec des toits d'ardoise, mais pendant les années précédant la Première Guerre, une rivalité apparaît entre les mouvements danois et allemand, chacun cherchant à montrer ce qu'il y avait de meilleur dans sa culture nationale respective.

21 Dans les premières années de cette rivalité, il n'y avait pas d'architectes de la tendance danoise dans le Schleswig du Nord. Le mouvement en faveur de l'architecture danoise eut donc recours aux architectes du «vieux» Danemark, en particulier ceux qui étaient 
associés au mouvement folklorique pour les lycées « Grundtvig ». Cette importation de la culture danoise est particulièrement bien représentée par les églises libres des villes de Haderslev (Hadersleben), Aabenraa (Apenrade) et Skærbæk (Scherrebek), ou par les grandes halles publiques comme le Folkehjem - la maison du peuple - à Aabenraa et le Sønderborghus de Sønderborg (Sonderburg). C'est ainsi que quelques-uns des plus grands noms de l'architecture du Danemark ont pu laisser leur empreinte dans le Schleswig du Nord, notamment Martin Nyrop (1849-1921), auteur du célèbre hôtel de ville de Copenhague $^{23}$.

22 (fig. 7)

Figure 7

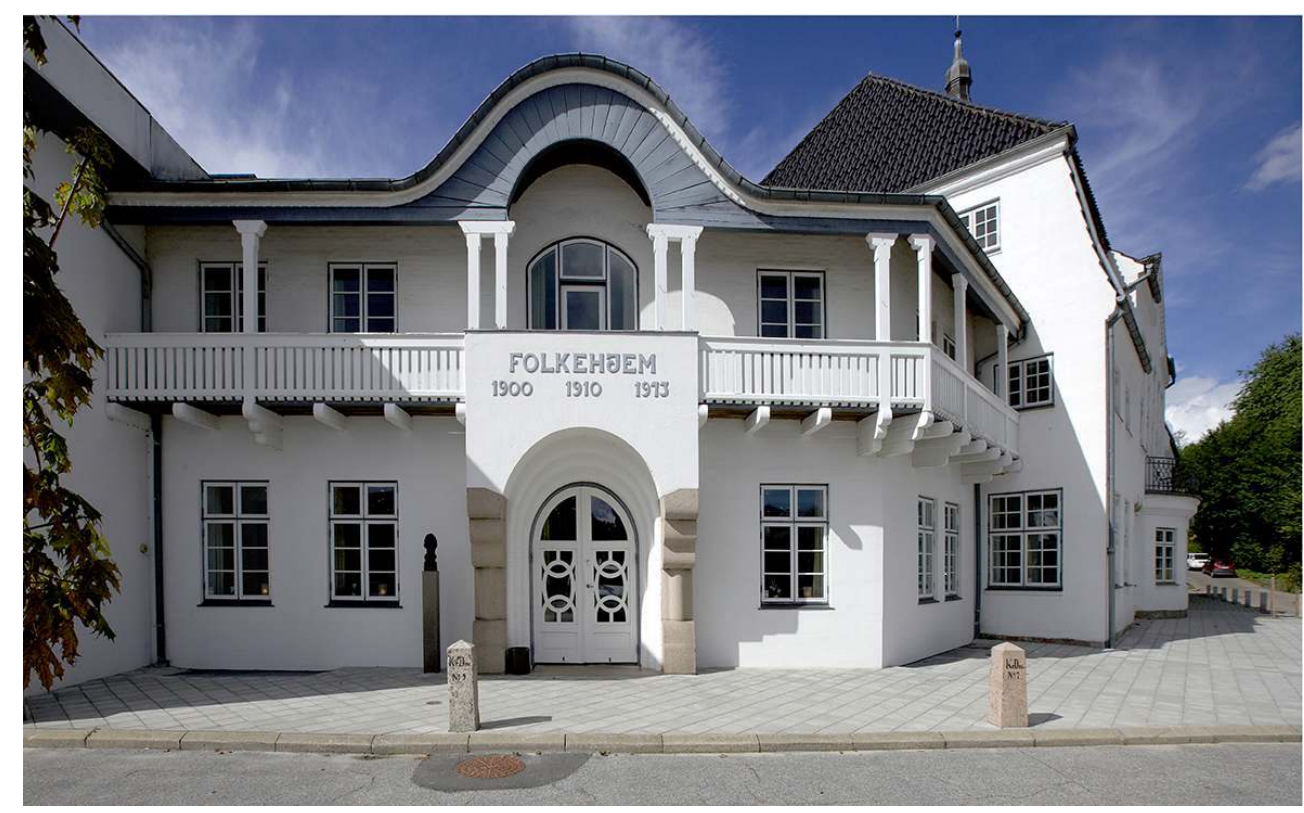

Le Folkehjem, halle d'assemblée, à Aabenraa, construit en 1910 par l'architecte Johannes Magdahl Nielsen (1862-1941), une manifestation de la force du mouvement danois dans le Schleswig du Nord, splendide exemple du romantisme national de Copenhague.

Phot. Søren Petersen. (c) Søren Petersen.

À la même époque toutefois, une nouvelle génération de jeunes architectes danois émerge, liés au mouvement de réforme et opposés à leurs aînés du romanticisme national. À la recherche d'inspiration pour un renouveau des traditions danoises, deux de ces architectes visitèrent le Schleswig du Nord en 1909, 1911 et 1912. Ils y entreprirent une campagne d'inventaire des vieux bâtiments des marais, comportant des études et des mesures précises. La nouvelle familiarité avec des traditions constructives préindustrielles du Schleswig de l'Ouest est à l'origine d'un nouveau style vernaculaire danois, appelé Bedre Byggeskik, c'est-à-dire "meilleure construction", du nom d'une association fondée pour promouvoir la tradition vernaculaire danoise. L'un des romanticistes nationaux a ironisé au sujet de cette révolution parmi les étudiants de l'Académie Royale : «Il semblerait qu'un nouveau slogan mobilise nos jeunes architectes, “À bas l'Italie, Hourrah pour Møgeltønder". » L'inspiration donnée par Møgeltønder eut ainsi un impact considérable non seulement sur l'architecture du Schleswig-Holstein mais plus largement, sur le développement d'une nouvelle architecture danoise. Le style Møgeltønder fut utilisé par les architectes de la tendance danoise dans le Schleswig du 
Nord, et certains des jeunes architectes qui avaient réalisé les inventaires purent avoir des commandes de la part de clients dans le Schleswig du Nord ${ }^{24}$.

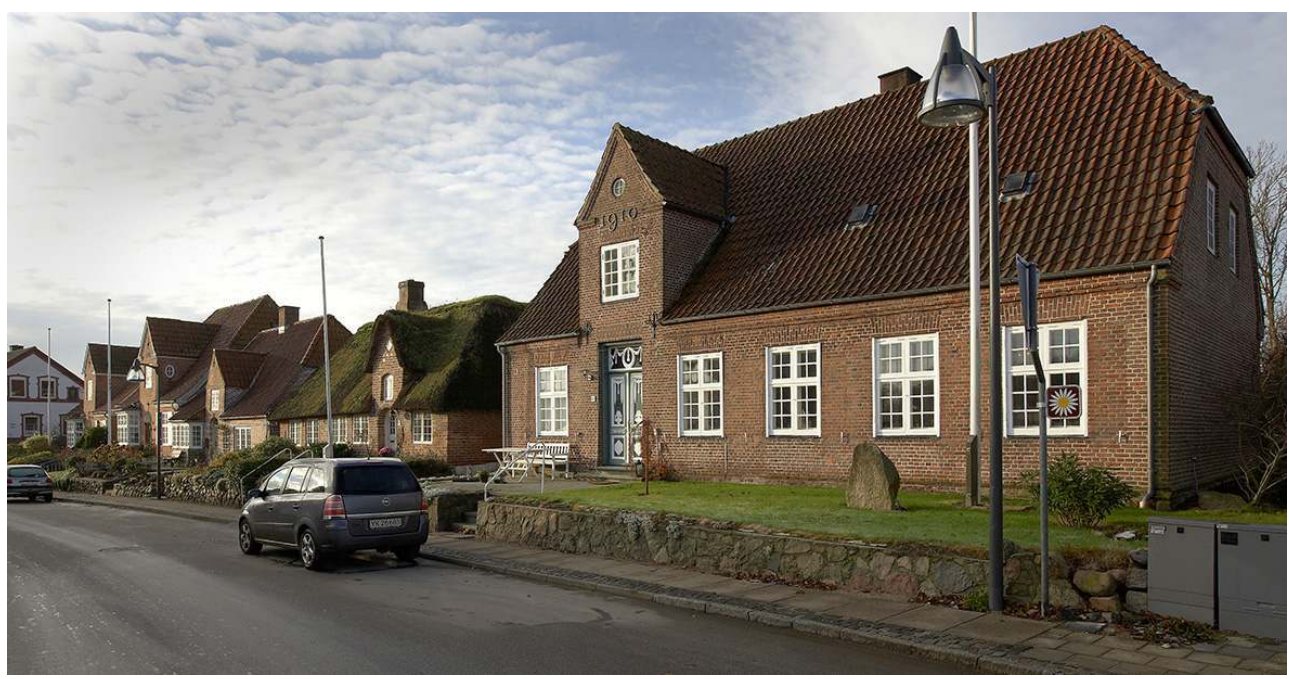

À Møgeltønder, H. C. Davidsen, le régisseur du domaine, de tendance danoise, a créé une série de belles maisons dans la tradition locale. À droite, sa première maison de 1910, ensuite deux maisons du XVIII' siècle, puis, à gauche, deux maisons de Davidsen dans le même style, construites en 1923, après la réunification avec le Danemark.

PHOT. SøREN PETERSEN. ( $)$ SøREN PETERSEN.

De cette manière, deux styles de construction vernaculaires ayant le Møgeltønder comme modèle commun firent leur apparition au Danemark et dans le Schleswig-Holstein, utilisé sans distinction par des bâtisseurs de tendance danoise ou allemande. Mais en dépit d'une pensée architecturale apparemment identique, le style Møgeltønder revêtit des significations très différentes pour les deux parties de la province. Pour le SchleswigHolstein allemand, le nouveau style vernaculaire incarnait la possibilité d'une cohabitation future, apaisée, multi-ethnique et multiculturelle, libérée des conflits nationaux mais en même temps, dissociée d'une domination culturelle prussienne tout en restant à l'intérieur de l'État germanique. L'investissement allemand dans de nouveaux bâtiments et de nouvelles institutions dans le Schleswig du Nord peut être vu comme une offensive culturelle après l'échec des politiques répressives à l'égard du mouvement danois, avec ses tentatives de colonisation, ses expulsions et ses incarcérations. Mais du point de vue danois, en revanche, le vernaculaire de style Møgeltønder était la preuve de l'appartenance ancienne de la région à la culture et à l'histoire danoises, une synthèse entre cultures régionale et nationale, même si, en réalité, les vieux bâtiments eux-mêmes résultaient d'influences culturelles en provenance de la Frise, des Pays-Bas et des régions de l'ouest du Danemark.

Après la division du Schleswig, à la suite du référendum de 1920, les deux versions du style vernaculaire Møgeltønder ne soulevèrent aucun problème ni d'un côté ni de l'autre de la nouvelle frontière. Après la "réunion", les autorités danoises investirent de manière importante dans la construction d'écoles, de lycées, d'églises et de salles communales, tandis que les réformes agraires nécessitaient également l'établissement de nouvelles fermes. Dans la plupart des cas, ces nouveaux bâtiments adoptèrent le 
vernaculaire Møgeltønder, mais dans quelques cas d'institutions, d'autres styles régionalistes purent être retenus, spécifiques, pour les îles à l'est de Sjaelland ou Funen. Un exemple : la nouvelle église de Rinkenæs, construite dans les années 1930 à proximité des eaux frontalières du fjord de Flensborg. Son dessin fut critiqué par des membres de la minorité germanophone car l'édifice imprimait « une marque du style Funen sur notre région ", où l'emploi de pignons à gradins et de couvertures en tuiles n'appartenait pas aux traditions médiévales locales.

(fig. 9)

Figure 9

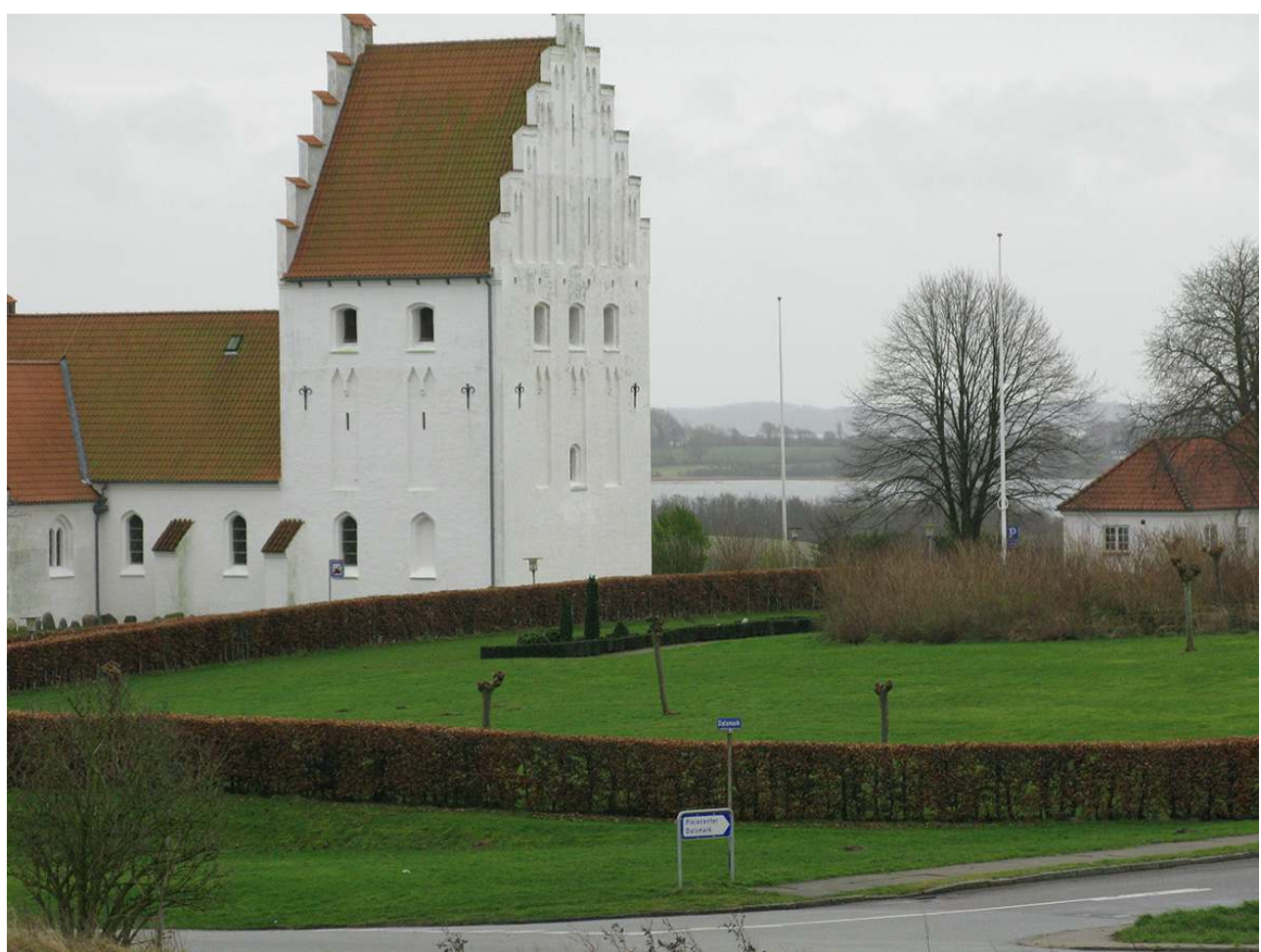

La nouvelle église paroissiale de Rinkenæs, à côté du fjord de Flensburg, a été construite de 1929 à 1932 suivant les plans de l'architecte Harald Lønborg Jensen (1871-1948), de Copenhague. II s'agissait d'une manifestation danoise dans une zone de nationalités mixtes, et comprise comme telle par la population locale allemande.

PHOT. PETER DRAgSBO. (C) PETER DRAgSBO.

Les autorités danoises avaient bien conscience que le territoire recouvré représentait encore une zone litigieuse, en raison de sa minorité allemande qui n'acceptait pas la frontière de 1920. Dès lors, elles furent soucieuses de montrer ce que la culture danoise avait de meilleur. De 1920 à 1940, l'architecture de cette région frontalière se caractérise par des valeurs nationales traditionnelles, sans aucune trace de modernisme danois, qui ne perce qu'après la Deuxième Guerre mondiale ${ }^{25}$. Au sud de la frontière, dans la province allemande du Schleswig, les autorités reconnurent également le statut particulier de la région frontalière et de nombreux projets de développement y virent le jour, surtout dans la ville encore contestée de Flensburg. Au centre de cette ville, une grande halle de réunion fut érigée, baptisée la Deutsches Haus (maison allemande) et surnommée le Reichsdank für deutsche Treue, soit « la gratitude du pays pour la loyauté allemande » car en 1920, la majorité des habitants de cette ville avaient voté pour l'Allemagne. L'édifice 
représente une combinaison de styles régionalistes, avec des éléments de l'expressionnisme allemand des années 1920 ; la tour de l'horloge, par exemple, est inspirée de celle de la célèbre gare ferroviaire de Stuttgart ${ }^{26}$.

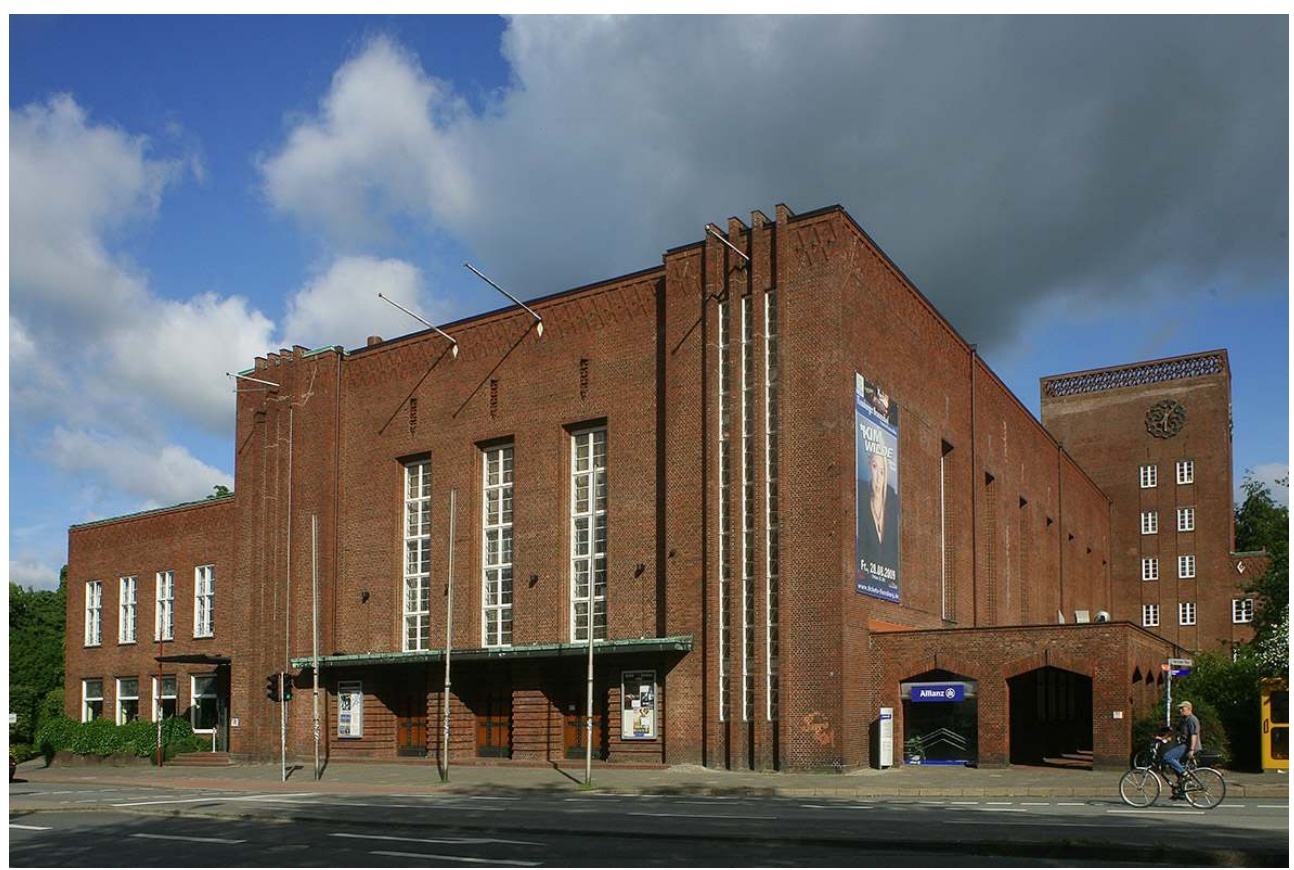

Cette « Deutsches Haus » de Flensburg a été construite de 1928 à 1930 comme un cadeau de la nation allemande à la Ville, qui avait voté majoritairement en faveur de l'Allemagne au référendum de 1920.

Phot. Denkmalamt Flensburg. (C) Denkmalamt Flensburg.

La division du Schleswig a créé deux minorités nationales qui ont désormais le droit d'établir leurs propres institutions et leurs propres écoles. Pour les deux parties de la région, l'usage du style vernaculaire du Schleswig reste une évidence car les minorités sont particulièrement attachées à la région, les Allemands s'appelant les Nordschleswiger (les gens du Schleswig du Nord) et les Danois les Sydslesvigere, les gens du Schleswig du Sud. La minorité danoise continua à utiliser le style vernaculaire régional jusque dans les années 1950. En revanche, la minorité allemande, comme l'ensemble de la République fédérale, occupée à rétablir toutes ses institutions après la Deuxième Guerre mondiale, se tourna vers le modernisme, symbole des valeurs démocratiques de l'Europe de l'aprèsguerre et d'une rupture avec le passé.

\section{Lorraine et Alsace, confrontation et concorde}

Après la défaite de la France dans la guerre franco-prussienne de 1870-1871, l'Alsace et une partie de la Lorraine furent annexées et incorporées dans le nouvel Empire allemand comme Reichsland Elsass-Lothringen (Pays d'Empire d'Alsace-Lorraine). Ce nom jumelé bien familier tend à faire oublier qu'en réalité, les deux régions étaient, et demeurent, très différentes. Leur histoire et leur architecture sont peut-être bien connues des lecteurs de ce numéro de la revue In Situ mais je vais néanmoins rendre compte ici des relations, dans 
les deux régions, entre nationalité et architecture. Ces relations présentent des parallèles intéressants avec celles du Schleswig, mais aussi quelques différences notables.

En 1871, la Lorraine, quoique bilingue, était caractérisée surtout par sa culture française et un état d'esprit français. Pendant l'occupation allemande, un fort mouvement de résistance fut organisé par le Lothringer Block. Après l'accession au trône de l'empereur Guillaume II, en 1888, on constate une "nationalisation » progressive des politiques culturelles et architecturales, avec une volonté de germanisation des territoires «peu sûrs » à la frontière. En Lorraine, la ville de Metz, à cent pour cent francophone, fut érigée comme «forteresse allemande de l'Ouest». Autour de 1900, la ville bénéficia d'un immense effort pour la création d'une cité allemande moderne, la Neustadt, élevée dans la zone libérée par la démolition des anciennes fortifications ${ }^{27}$.

\section{Figure 11}

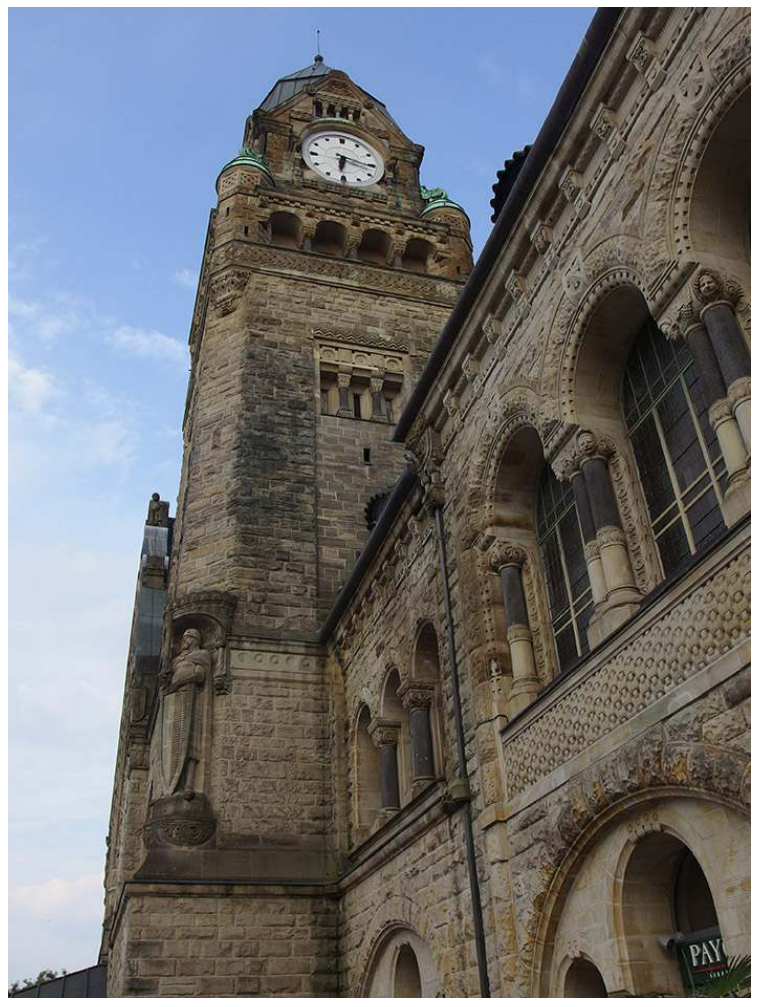

La gare principale de Metz, construite de 1903 à 1905, point central de la Neustadt allemande. Le projet architectural d'origine fut remodelé suivant les désirs personnels de l'empereur Guillaume II pour imposer le style impérial « Hohenstaufen».

PHOT. PETER DRAgSBO. (C) PETER DRAgSBo.

À Metz, Guillaume II s'engagea personnellement dans les politiques architecturales et, sous son influence impériale, la gare principale, l'hôtel des postes et la nouvelle cathédrale protestante furent construits dans le style roman "Hohenstaufen ». Ces manifestations matérielles d'autorité relevaient d'une volonté plus générale d'imprimer une marque allemande sur la Lorraine. L'usage d'un régionalisme alsacien ou rhénan fut critiqué par les architectes français comme une "rhénanisation». L'architecture ancienne, en Lorraine, étant dominée par les traditions françaises, les tentatives 
allemandes de légitimation par une architecture régionaliste ne trouvèrent pas beaucoup de fondement ${ }^{28}$.

La réponse française à ce nationalisme architectural fut le développement d'une « contrearchitecture» inspirée de l'architecture haussmannienne de Paris, datant du renouvellement urbain sous le Second Empire. Cette architecture néo-baroque s'est maintenue à Metz jusque dans les années 1930, manifestant un conservatisme architectural que l'on peut constater dans d'autres régions « sur le front ».

Figure 12

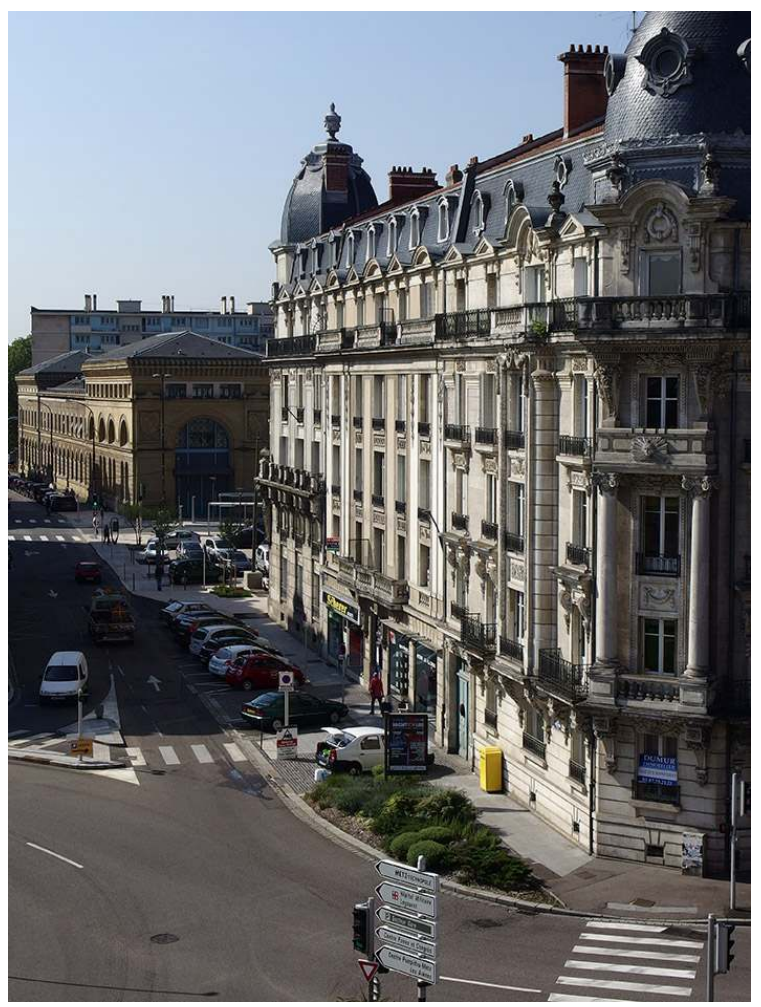

À Metz, le contre-style architectural français a été utilisé jusque dans les années 1920, après la réunification de la Lorraine à la France. Bâtiments datant des années 1920 place du Roi-George. Au fond, l'ancienne gare ferroviaire, datant de 1878.

Phot. Helle Ravn. ( ) Helle Ravn.

À la différence de la Lorraine, l'Alsace était caractérisée de longue date par sa langue alémanique et sa culture populaire germanique. Mais depuis le XvIII siècle, la province s'était montrée loyale envers la nation française et son idéologie nationale. Peu de temps après l'annexion de 1871, l'Allemagne commença à faire de Strasbourg (Strassburg) la capitale métropolitaine de l'Alsace-Lorraine, symbole de la puissance et de la civilisation allemande. Une ville entièrement nouvelle, la Neustadt, fut dessinée, dotée de grands boulevards, de vastes places et d'un palais impérial, d'une université, de bâtiments administratifs et d'églises. Ces bâtiments furent conçus selon les idéaux des grands styles européens comme la Renaissance italienne ou le baroque français, avec une poignée d'exemples de néogothique allemand. Même le palais impérial fut dessiné dans un style baroque inspiré du palais du Louvre à Paris, empruntant ainsi à l'« ennemi » le symbole de puissance ${ }^{29}$. 

fort soutien de la sociale démocratie considérée comme le meilleur moyen de s'opposer au pouvoir autoritaire de Berlin. L'Alsace fut marquée alors par un désir d'autonomie, exprimé par des figures comme l'écrivain René Schickelé. Ce mouvement continua à caractériser la vie politique alsacienne entre les deux guerres, le mouvement autonomiste cherchant dorénavant à s'affranchir du centralisme français. Du point de vue architectural, ce désir d'autonomie trouva son expression dans le développement d'un Heimatstil spécifique à l'Alsace, inspiré par les académies de Stuttgart et de Karlsruhe. Comme dans le Schleswig, les architectes des deux tendances, allemande et française, eurent recours à l'architecture vernaculaire alsacienne. Et comme dans le Schleswig, l'usage de ce style perdura jusqu'après la Deuxième Guerre mondiale, appliqué à la reconstruction de villages alsaciens après les destructions de la guerre. En même temps, l'architecture de Strasbourg fut dominée par le «um 1800 », une forme de classicisme ${ }^{30}$ développé dans les académies de Stuttgart et de Karlsruhe. Ce classicisme régional, loin des idéaux de l'Académie des beaux-arts en France, domina l'architecture publique et privée de Strasbourg jusqu'à la fin des années $1940^{31}$.

(fig. 13)

Figure 13

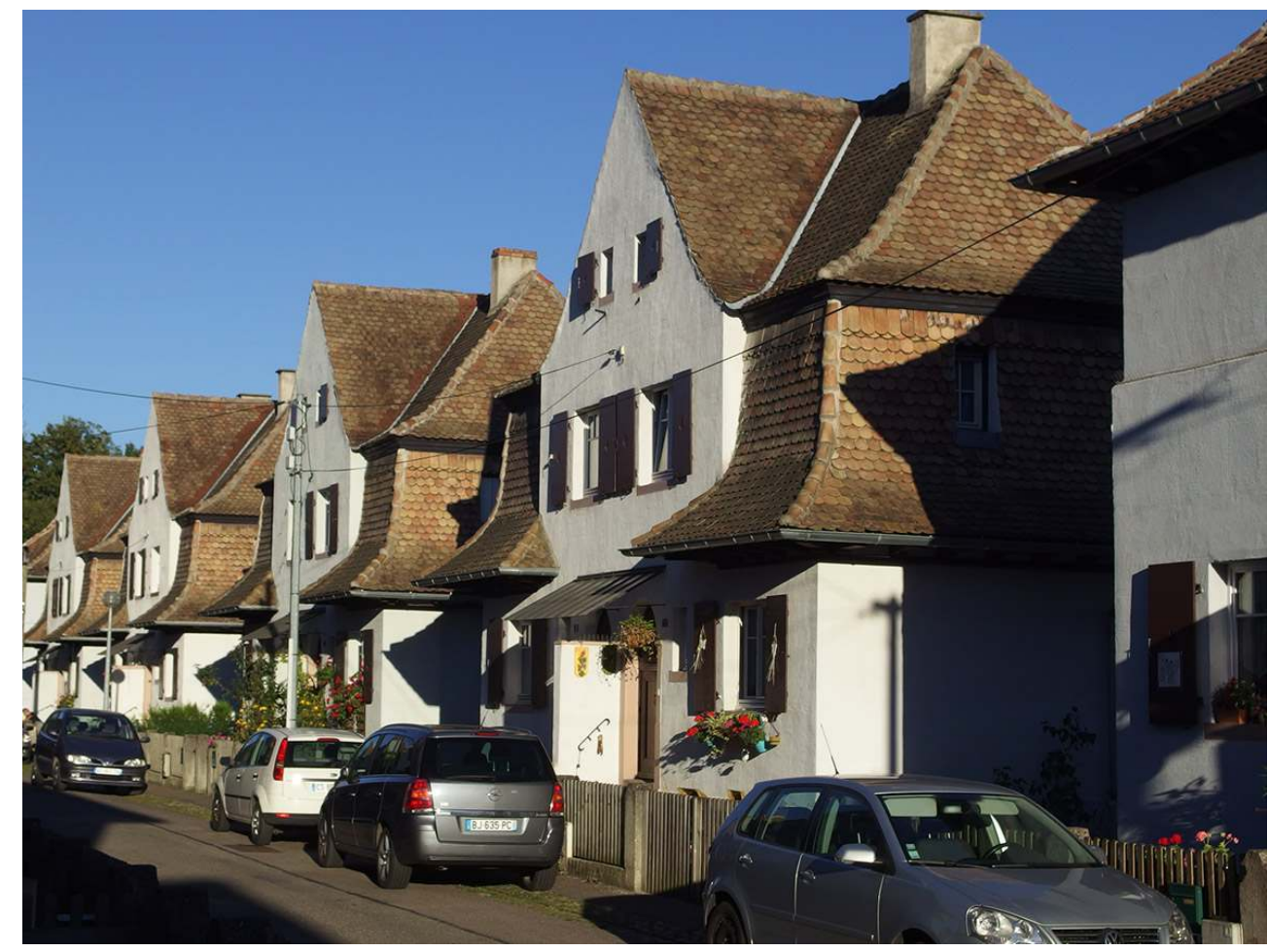

La cité-jardin de Stockfeld, construite à partir de 1910 pour des populations expulsées du centre-ville de Strasbourg à cause des destructions d'habitats insalubres. Le Stockfeld fut l'une des premières et des plus grandes cités-jardins allemandes et un témoin remarquable du style vernaculaire alsacien.

PHOT. PETER DRAgSBO. (C) PETER DRAgSbo.

La nature des conflits nationaux dans le Schleswig et en Lorraine présente ainsi de nombreux parallèles, les deux régions étant marquées par leurs mouvements irrédentistes. Mais en même temps, la «contre-architecture » empreinte de nostalgie parisienne, à Metz, n'a pas d'équivalent dans le Schleswig du Nord, où le mouvement pro- 
danois s'identifiait à un Danemark moderne et démocratique et à son architecture contemporaine. Et à la différence des autorités allemandes du Schleswig, il n'y eut aucune tentative, du côté français, ni avant ni après la « réunification » de 1918, de puiser dans les traditions régionales en architecture. La notion française d'un libre choix de nationalité, exprimée par l'auteur Ernest Renan (1823-1892)32, était une question avant tout de loyauté envers la nation. Les autorités ne voyaient pas dès lors le besoin d'une légitimation par l'usage d'un régionalisme architectural. En ce qui concerne l'Alsace, le consensus architectural sur le style régional vernaculaire trouve un parallèle dans le Schleswig, mais à la différence de cette dernière région, il n'y eut pas d'interprétation spécifiquement française ou allemande de ce régionalisme. Et si la Lorraine, comme le Schleswig, est restée partagée entre des loyautés envers deux mères-patries, l'Alsace quant à elle a vu naître un désir d'autonomie, mal compris tant par les autorités françaises qu'allemandes.

\section{La Prusse-Occidentale et Poznań, autres champs de bataille} par un autre point de vue qui contraste avec elles. C'est pour cette raison que j'ai souhaité inclure ici l'étude d'autres territoires frontaliers, en Prusse-Occidentale et dans le Tyrol du Sud. Ces deux exemples suggèrent, à juste titre, qu'un mouvement de résistance nationale ne s'intéresse pas nécessairement à des questions d'architecture, même si les autorités de gouvernement, elles, utilisent l'architecture de manière démonstrative. furent annexés par la Prusse en 1772-1775, lors de la partition de la Pologne entre la Prusse, l'Autriche et la Russie. C'est ainsi que la partie prussienne du territoire polonais était non seulement une région frontalière mais aussi une fraction importante d'un ancien royaume, dominée, grosso modo, par la langue et l'ethnicité polonaises. Des villes comme Cracovie et Poznań (Posen) devinrent des centres importants de mouvements de résistance nationale. Par conséquent, il était très difficile de déclarer que la PrusseOccidentale et la province de Poznań étaient des territoires de «culture allemande ancienne ", comme les Allemands ont pu le faire pour le Schleswig ou l'Alsace-Lorraine. Tout au long du xix siècle, il y eut une lutte incessante pour la propriété des terres et la domination culturelle, et la ville de Poznań fut un vrai champ de bataille entre les deux nationalités. L'inauguration d'un théâtre polonais fut suivie de près par l'ouverture d'un théâtre allemand, à proximité ; face à la bibliothèque polonaise, on construisit le nouveau musée Kaiser Friedrich; la banque nationale allemande fut édifiée à côté de l'hôte polonais, et ainsi de suite ${ }^{33}$.

En raison de la très grande majorité de Polonais qui habitaient en Prusse-Occidentale, les autorités allemandes lancèrent en 1888 une campagne de colonisation massive accompagnée de mesures contre l'usage de la langue polonaise et contre l'Église catholique, vecteur important de l'identité nationale polonaise. Comme élément de cette politique "éducative », Poznań devint la "forteresse frontalière allemande de l'est ", à l'instar de Strasbourg et Metz à l'ouest. Cette décision comprenait la conception de toute une partie nouvelle de la ville, dessinée par le célèbre ingénieur civil Joseph Stübben (1845-1936). Au cœur de cette ville nouvelle, la pièce de résistance était le palais impérial 
dessiné par Franz Schwechten dans le style national Hohenstaufen utilisé pour les grands bâtiments de Metz ${ }^{34}$.

Figure 14

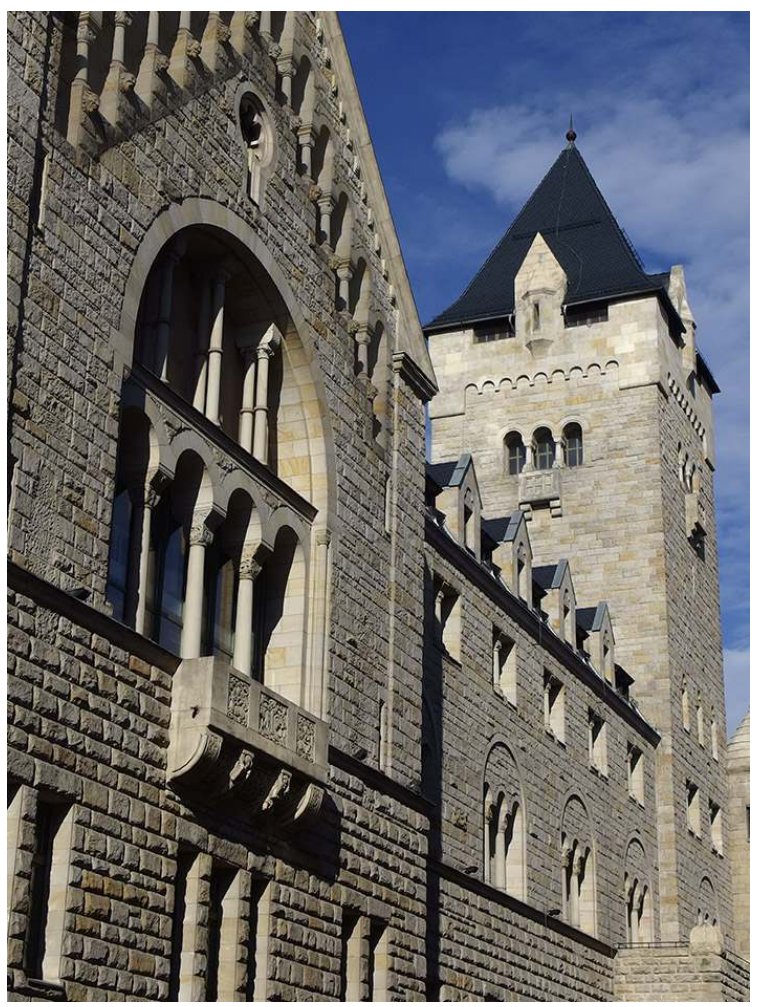

Le château impérial de Poznań était le point central de la Neustadt allemande et le symbole de la puissance allemande dans ces régions contestées de Pologne. II est dû à l'architecte préféré du Kaiser, Franz Schwechten, en 1905-1910.

PHOT. PETER DRAgSbo. (C) PETER DRAgSbo.

En raison de l'absence d'histoire et de culture folklorique allemandes en PrusseOccidentale, les Allemands ne s'efforcèrent pas de concevoir un régionalisme allemand particulier. L'architecture allemande de Poznań et des provinces environnantes fut essentiellement influencée par l'historicisme prussien ou berlinois, le Jugendstil ou le märkisch néogothique. Les programmes pour implanter une population allemande et la répression de l'usage de la langue et de la religion polonaises montrent toutefois que les autorités allemandes n'étaient pas indifférentes à la question d'ethnicité.

Du côté polonais, en opposition aux projets nationaux allemands, d'autres domaines furent préférés à l'architecture comme expression de la lutte nationale. Il n'y eut donc pas de tentatives de produire une contre-architecture polonaise. La lutte se concentra davantage sur l'installation d'institutions polonaises dans la ville, la question de la propriété foncière à la campagne, et, plus généralement, sur le combat pour l'identité polonaise dans la langue, la littérature, la religion et la politique ${ }^{35}$.

47 Après la résurrection de la Pologne en 1918, le gouvernement n'eut pas de politique architecturale clairement affirmée, cherchant, d'un côté - et à Varsovie en particulier - à démontrer que le pays appartenait dorénavant à une Europe moderne et souhaitant, de l'autre côté, développer une architecture polonaise authentique, fondée sur l'histoire et le 
patrimoine et centrée sur Cracovie et Poznań. Ainsi, les architectes de Poznań mobilisèrent-ils différentes variétés du baroque polonais ou du classicisme pour les bâtiments élevés à l'occasion de la grande exposition de Poznań de 1929, organisée pour commémorer les dix ans de l'indépendance du pays. Comme en France, le classicisme apparaissait comme le symbole de la culture "latine», associée, en Pologne, au catholicisme, en opposition au «monde germanique ». Ce néo-classicisme polonais était proche, toutefois, du développement contemporain du néo-classicisme en Allemagne, dans les pays scandinaves et bien d'autres ${ }^{36}$.

Figure 15

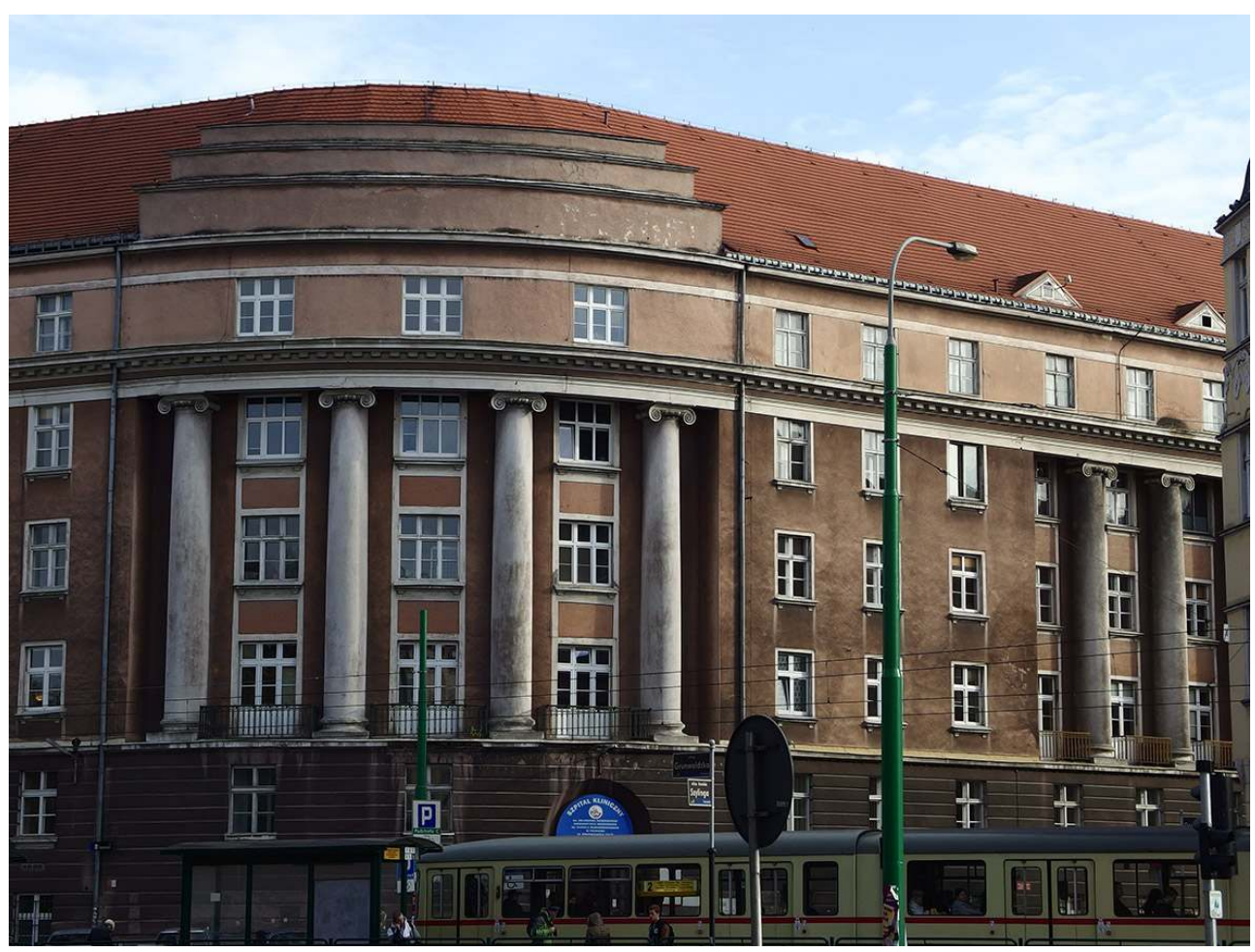

Après 1918, l'architecture polonaise a hésité entre le romantisme national, le baroque et le modernisme. Comme champ de bataille national, Poznań était caractérisé par une conception conservatrice de l'architecture, illustrée ici par l'ancien hôtel Polonia, construit pour l'Exposition de 1929.

Phot. Peter Dragsbo. (C) Peter Dragsbo.

\section{Tyrol du Sud}

Comme on l'a dit, nous incluons le Tyrol du Sud (Südtirol) dans cette analyse à la fois pour voir le cas d'une région frontalière en dehors de l'Empire allemand et en raison de la nature du conflit national que cette région a connu, des événements plus récents et plus durs. L'annexion par l'Italie du Tyrol du Sud en 1919-1920 fut une des conséquences de la défaite de l'Autriche-Hongrie dans la Première Guerre mondiale. Avant la guerre, toutefois, cette région ne connaissait aucun conflit de nature nationaliste: les revendications italiennes sur la région étaient très récentes et la région ne comportait aucune minorité italienne. La population Ladiner, qui parlait ladin, ne se considérait pas 
comme italienne. La plupart des noms de lieux italiens du Tyrol du Sud ont été inventés par Ettore Tolomei (1865-1952) au tournant du siècle. Ici, c'est tout le contraire de la situation du Schleswig, où des noms de lieux en allemand existaient depuis les $\mathrm{XV}^{\mathrm{e}}$ et $\mathrm{XVI}^{\mathrm{e}}$ siècles.

Figure 16

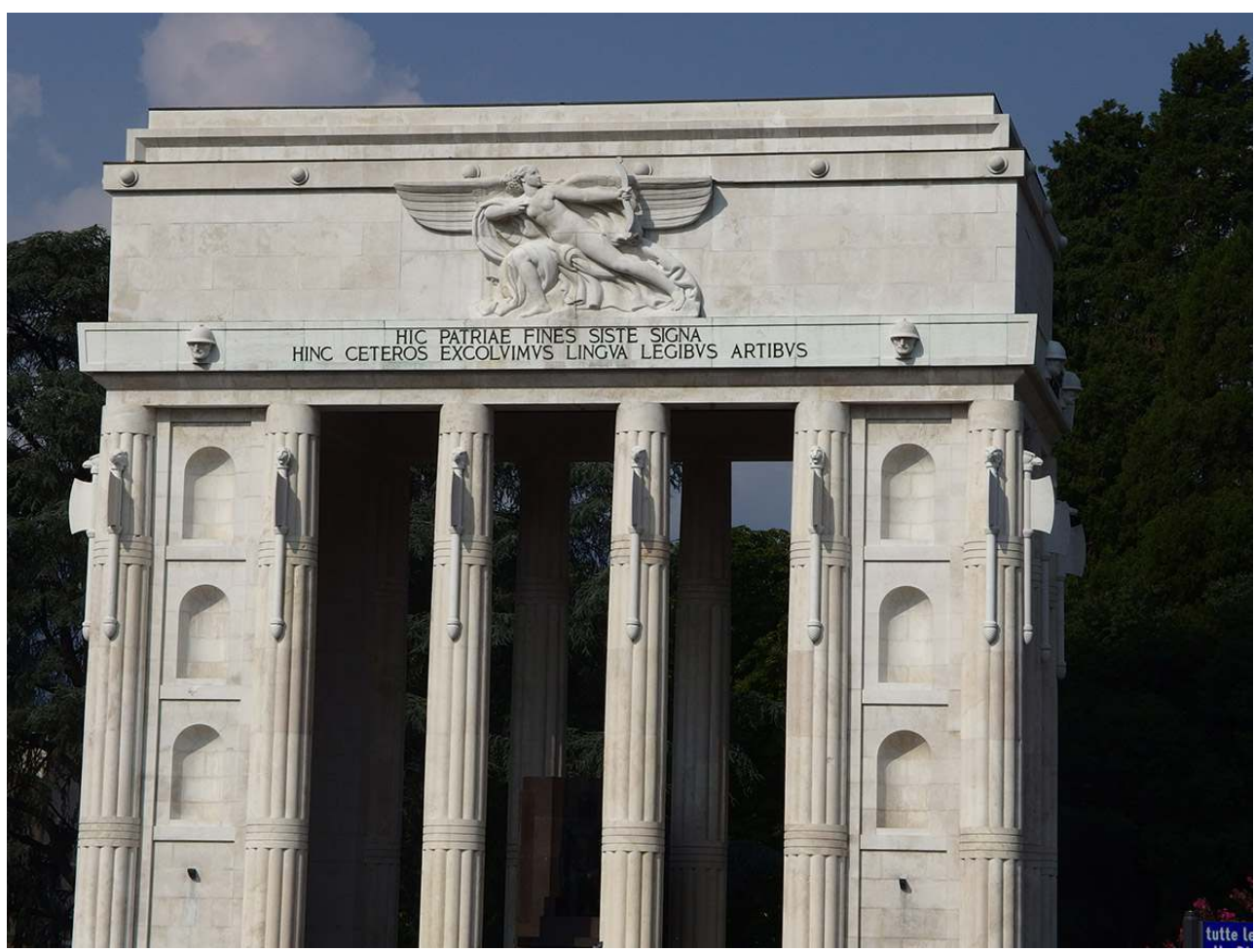

Le monument à la Victoire italienne de Bozen (Bolzano) fut conçu comme un arc de triomphe romain. L'inscription en latin peut être traduite par : «Ici se dresse la frontière. Depuis ce point, nous avons éduqué l'autre grâce à la langue, la loi et la culture ». À l'origine, « l'autre » devait se lire « les barbares ». Phot. Peter Dragsbo. (c) Peter Dragsbo.

51 À la suite de l'annexion, les nouvelles autorités italiennes essayèrent très rapidement d'imprimer une forte marque italienne sur le territoire, en particulier dans la capitale régionale de Bozen (Bolzano), en y introduisant l'architecture traditionnelle de la région de Vénétie. Après la prise du pouvoir par Mussolini, en 1922, un plan ambitieux de transformer Bolzano en cité modèle du fascisme fut mis en place, comprenant la construction d'un monument à la victoire sous forme d'un arc de triomphe et tout un nouveau quartier de la ville conçu selon le style fasciste, avec des arches romaines, des places officielles comme la piazza Vittoria et des bâtiments pour le mouvement fasciste, la police et l'armée. Mais à côté de tels bâtiments en marbre et brique, les édifices destinés aux sports et à la jeunesse étaient d'un style très moderne, comme le quartier général pour la Gioventù Italiana del Littorio (G.I.L., la Jeunesse fasciste) ou le stade Drusus. 
Figure 17

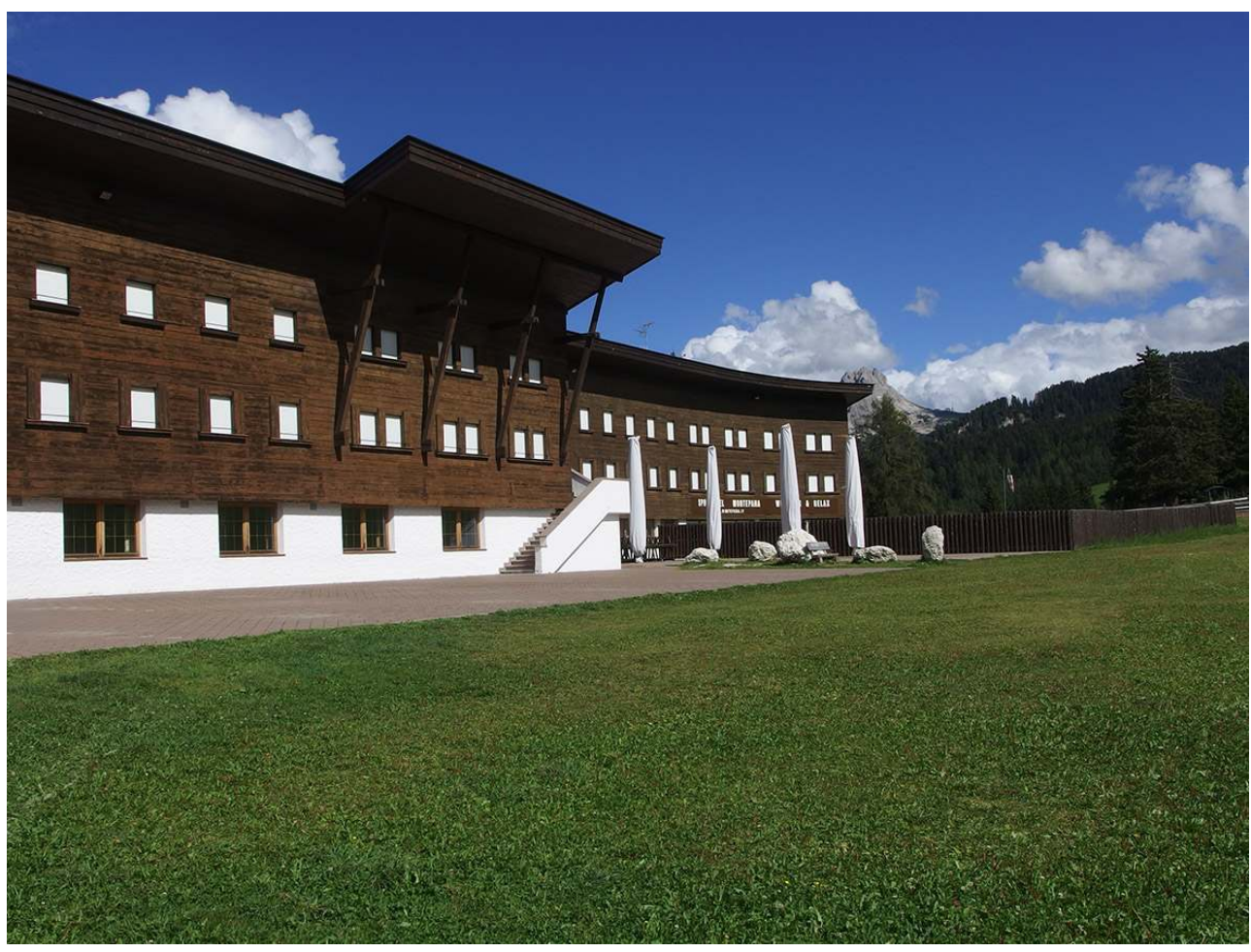

Un bon exemple du style «Tiroler Moderne », cet hôtel de montagne à Monte Pana dans les Dolomites, dessiné par l'architecte d'Innsbruck Franz Baumann (1892-1974) en 1931.

Phot. Peter Dragsbo. (C) Peter Dragsbo.

En raison de la suppression radicale de la culture autrichienne et tyrolienne, aucun architecte de la région ne put obtenir de commande de bâtiments. Le Tyrol du Sud dut chercher ses architectes en Autriche, et notamment à Innsbruck. Une école architecturale se développa ici, appelée Tiroler Moderne, comportant un mélange audacieux de modernisme et de matériaux traditionnels. C'est ce style qui fut importé au Tyrol du Sud. Par conséquent, dans cette région, il n'y eut aucun emploi d'architecture vernaculaire, même si la maison traditionnelle tyrolienne était bien connue à travers l'Europe. Après la Deuxième Guerre mondiale, lorsque les espoirs tyroliens d'autonomie ou de réunification avec l'Autriche furent anéantis, le mouvement de résistance se focalisa sur les grandes centrales hydro-électriques italiennes, qui furent l'objet de tentatives de sabotage jusque dans les années $1970^{37}$.

\section{L'architecture des régions frontalières, un patrimoine encombrant?}

Étant donné la tendance générale à "nationaliser » le concept de "patrimoine », notre architecture des régions frontalières présente un double défi. Il y a tout d'abord le risque d'ignorer les cultures et les identités spécifiques de ces régions frontalières, obnubilées par l'effort d'établir une unité nationale. Dans leurs régions frontalières, des pays sont souvent confrontés à des traces de " quelque chose d'autre ", parfois liées à des souvenirs désagréables d'annexion ou d'occupation, de lutte nationale et de répression, de conditions politiques difficiles, etc. ${ }^{38}$. Le patrimoine allemand, dans ces régions 
frontalières européennes, en particulier le style impérial lourd de symboles, ou le romanticisme national prussien, représente un défi durable pour les politiques nationales du patrimoine au Danemark, en France et en Pologne. Les historiens et les responsables du patrimoine doivent souvent se placer à l'avant-garde en termes de reconnaissance et d'acceptation, le grand public étant généralement moins enthousiaste.

À mes yeux, il existe un lien évident entre l'acceptation de ce patrimoine germanique et la détente politique en Europe. La France offre ici un exemple intéressant et précurseur. La réconciliation avec l'Allemagne, en dépit de l'inimitié séculaire des deux pays, a entraîné une coopération étroite dans le cadre de la Communauté européenne. Des mesures de protection du patrimoine allemand en Alsace et en Lorraine ont été prises dès les années 1970, comprenant, par exemple, le palais impérial (le palais du Rhin), à Strasbourg ${ }^{39}$. Plus récemment encore, en 2017, la Ville de Strasbourg a réussi à faire intégrer la Neustadt allemande dans sa zone inscrite au patrimoine mondial de l'Unesco ${ }^{40}$. En Pologne, une reconsidération du patrimoine allemand a pu s'amorcer après la chute du rideau de fer, mais l'acceptation de ce patrimoine est peut-être plus difficile en raison des conditions de vie très rigoureuses et des souvenirs douloureux des périodes d'occupation allemande. L'acceptation est arrivée d'abord dans les « anciennes » provinces allemandes de Prusse-Occidentale et de Poznań, dont le passé polonais ne faisait pas débat. Ailleurs, dans les régions annexées après la Deuxième Guerre mondiale, où l'ancienne histoire allemande est encore présente, ce passé et son patrimoine sont acceptés avec davantage de réticence ${ }^{41}$. Quant au Schleswig du Nord, l'attitude à l'égard du passé allemand a été plutôt négative, en dépit d'un processus apaisé de réconciliation entre Danois et Allemands dans ce territoire frontalier. Ici, la raison réside peut-être dans le fait qu'il n'y a pas eu de véritable occasion de reconsidérer le patrimoine multinational du Schleswig du Nord, étant donné le profond scepticisme des Danois à l'égard de l'Europe ${ }^{42}$.

Dans le Tyrol du Sud, enfin, le conflit national a existé jusqu'à une date récente, charriant des contradictions entre les autorités italiennes et la majorité germanophone, et également entre la majorité italienne dans la capitale, Bozen (Bolzano), et le gouvernement local. De fait, la région est riche de ce patrimoine qu'on a qualifié d'encombrant, y compris le monument à la victoire italienne et la frise monumentale qui décore la façade du bâtiment de l'administration des finances, construit à l'origine comme Casa del Fascio ou Casa Littoria, quartier général du mouvement fasciste ${ }^{43}$; cette frise acclame Mussolini comme un César victorieux. En 2014-2015, après de longs débats, la collectivité locale a réussi à transformer le monument à la victoire en monument commémoratif pour les victimes du fascisme et de la déportation. Mais le devenir de la frise mussolinienne est encore incertain ${ }^{44}$. 


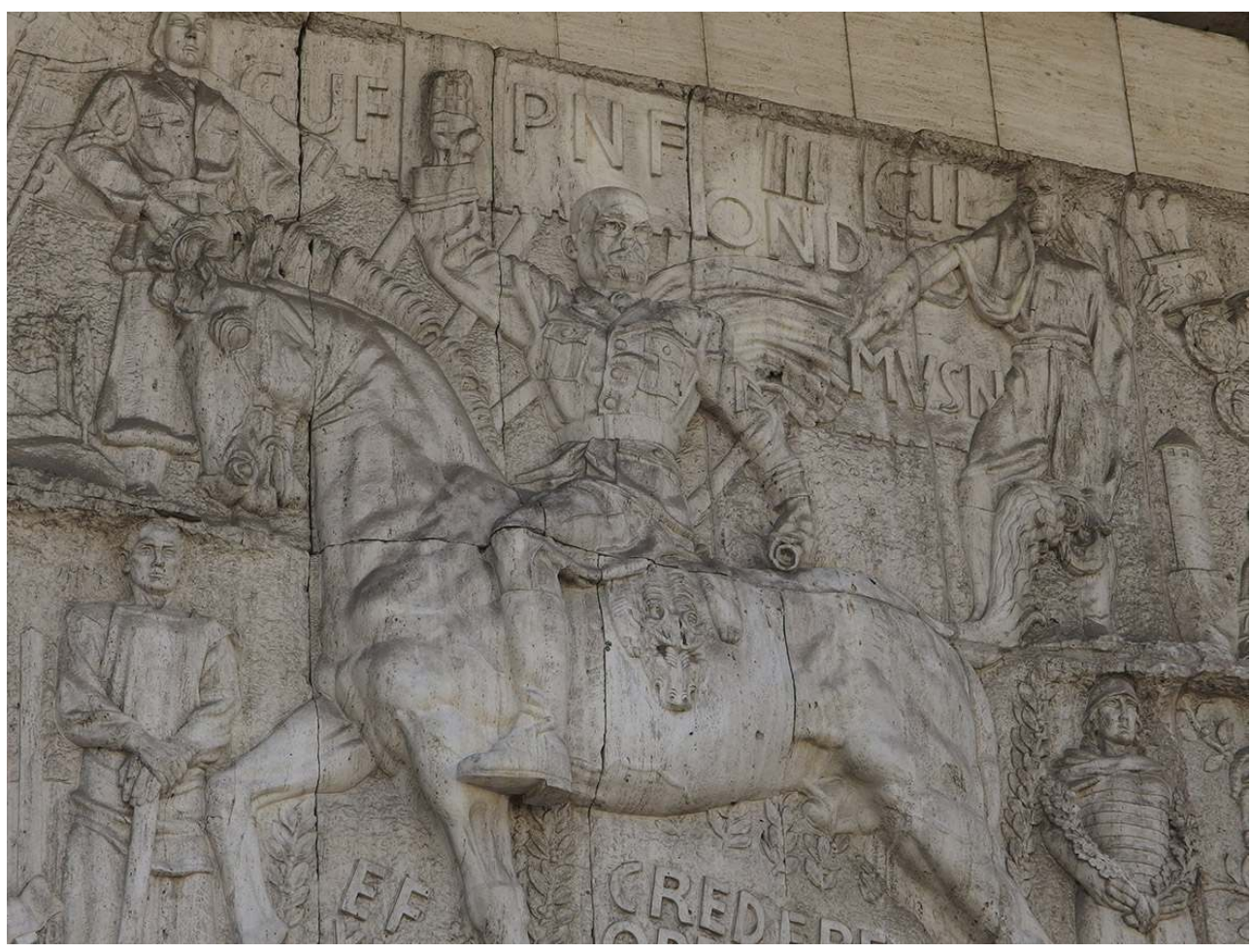

Patrimoine inconfortable : la frise géante qui décore la façade de l'ancien quartier général du parti fasciste à Bozen, construit en 1939 et occupé aujourd'hui par l'administration financière du Tyrol du Sud. Mussolini est montré en César conquérant, avec l'injonction, en italien, « Croire, Obéir, Combattre ».

PHOT. PETER DRAgSBO. () PETER DRAgSBo.

\section{Conclusion}

En regardant de nouveau les cinq régions frontalières analysées ici, on pourrait conclure que les relations entre nation et nationalité, d'un côté, et architecture, de l'autre, offrent d'intéressants exemples de similitudes et de différences. Le Schleswig et la Lorraine, par exemple, présentent des parallèles en ce qui concerne les divisions nationales et le développement de contre-architectures pour s'opposer aux politiques architecturales allemandes hégémoniques. Mais, à la différence du Schleswig, les architectes de la tendance française en Lorraine ont préféré un style nostalgique évoquant le «vieux Paris ». Dans le Schleswig du Nord, le mouvement danois est resté proche de l'architecture contemporaine danoise.

Cela dit, en Lorraine, aucune des deux parties n'a développé un style vernaculaire distinctif. Les parallèles entre le Schleswig et l'Alsace dans le développement d'un style vernaculaire sont ici plus frappants. Le style vernaculaire était accepté par tout le monde, mais le Heimatstil du Schleswig était encore considéré comme un style Schleswigallemand ou Schleswig-danois par les deux parties. En Alsace, le style vernaculaire était plutôt le vecteur d'un souhait assez répandu d'autonomie. Quant à Posen, à la PrusseOccidentale et au Tyrol du Sud, aucun des mouvements irrédentistes de ces régions ne se préoccupait d'architecture, misant sur d'autres domaines dans la lutte nationale, la langue, par exemple, la propriété foncière ou la présence publique. 
60 l'empereur Guillaume II et de son architecte Franz Schwechten, peuvent être vus comme une tentative de régler la question de la diversité historique et culturelle au sein du Reich. Toutefois, les manifestations du style impérial furent plus particulièrement réservées aux «forteresses frontalières» de Metz et de Poznań. Niels Wilcken a interprété ce phénomène comme l'expression d'une revendication de pouvoir plutôt mal fondée, dans des régions frontalières à la vie publique divisée ${ }^{45}$.

61 En dépit de toutes les frontières et des divisions entre les nations, il ne faut pas négliger le très fort impact transnational de grands courants de l'architecture européenne ni l'influence des académies d'architecture. Le régionalisme de l'Allemagne du Sud a pu étendre son influence depuis l'académie de Munich à toute la Bavière mais également en Autriche, dans le Tyrol et en Alsace. Autre exemple : le néo-classicisme de Strasbourg des années avant et immédiatement après la Première Guerre mondiale. Développé d'abord dans les académies de Stuttgart et de Karlsruhe, ce style a conservé sa prééminence de chaque côté du Rhin jusqu'à la Deuxième Guerre mondiale. De la même manière, le Heimatstil du Schleswig, amalgamé avec le style Bedre Byggeskik danois, fut utilisé partout au Danemark jusqu'après la Deuxième Guerre.

Pour conclure, et pour reconnaître cette architecture frontalière comme patrimoine, il faut souligner ici la responsabilité de chaque nation envers le patrimoine situé à l'intérieur de ses frontières, quelle que soit l'identité des créateurs de ce patrimoine. On pourrait même dire que la conscience de l'importance de ce patrimoine «venu d'ailleurs » devrait être plus grande encore. Le patrimoine «étranger» est vital pour souligner des aspects de la culture et du récit national, facilitant la compréhension des différents protagonistes. Les chercheurs, les conservateurs du patrimoine et les historiens partagent la responsabilité de transformer les patrimoines nationaux en un patrimoine commun $^{46}$.

\section{NOTES}

1. - DRAGSBO, Peter. Arkitektur til grcensen. Arkitektur og nation i europæeiske grcenselande 1850-1940. Sønderborg: Museum Sønderjylland, 2014 ; DRAGSBO, Peter. «Architektur und Nation in Schleswig - und anderen europäischen Grenzgebieten ». Dans TRAGBAR, Klaus (éd.). Innsbrücker Beiträge zur Baugeschichte 1, Berlin/Munich : Deutscher Kunstverlag 2019, p. 10-29.

2. - MILLER LANE, Barbara. National Romanticism and Modern Architecture in Germany and the Scandinavian Countries. Cambridge/New York : Cambridge University Press, 2000.

3. - WILCKEN, Niels. Architektur im Grenzraum. Das öffentliche Bauwesen im Elsass-Lothringen 1871-1918 . Sarrebruck : Institut für Landeskunde im Saarland, 2000.

4. - KÖSTER, Bernd (éd.). GrenzWerte. Baukultur in Süd-Dänemark und Schleswig-Holstein / Grcensevcerdier Bygningskultur i Syddanmark og Slesvig-Holsten 1912-2012-2112. Handewitt : Leupelt, 2013.

5. - COHEN, Jean-Louis et FRANK, Hartmut (éd.). Interférences / Interferenzen. Architecture Allemagne France, 1800-2000. Cat. exp., Strasbourg, musée d'Art moderne et contemporain, Francfort-sur-leMain, Deutsches Architekturmuseum. Tübingen : Ernst Wasmuth, 2013.

In Situ, 38 | 2019 
6. - PIGNON-FELLER, Christiane. Metz. 1848-1918. Les métamorphoses d'une ville. Paris : Éditions du patrimoine, 2013.

7. - FRANZ, Birgit, DOLFF-BONEKÄMPER, Gabi et MAIER, Hans-Rudolf (éd.). Grenzverschiebungen, Kulturraum, Kulturlandschaft. Kulturerbe in Regionen mit wechselnden Herrschaftsansprüchen. Holzminden : Mitzkat, 2009.

8. - MIGNOT, Claude. L'Architecture au XIX ${ }^{e}$ siècle. Paris/Fribourg : Éditions du Moniteur/Office du Livre, 1983, et MORAVÁNSZKY, Ákos. Die Architektur der Donaumonarchie, 1867 bis 1918. Budapest/ Berlin : Corvina/Ernst und Sohn, 1988.

9. - MILLER LANE, Barbara. Op. cit., p. 207-48.

10. - APPLEGATE, Celia. A Nation of Provincials. The German Idea of Heimat. Berkeley : University of California Press, 1990.

11. - MAKAŚ, Emily Gunzburger et CONLEY, Tanja Damljanovic (éd.). Capital Cities in the Aftermath of Empires. Planning in Central and Southeastern Europe. Londres: Routledge, 2009, et ALOFSIN, Anthony. When Buildings Speak. Architecture as Language in the Habsburg Empire and its Aftermath. Chicago : The University of Chicago Press, 2006.

12. - Parmi les six frontières arrêtées par référendum, suivant les principes du président Woodrow Wilson, seules les frontières entre l'Autriche et la Hongrie, l'Autriche et la Slovénie et l'Allemagne et le Danemark sont encore en place de nos jours. Voir JEBSEN, Nina. Als die Menschen gefragt wurden. Eine Propagandaanalyse zu Volksabstimmungen in Europa nach dem Ersten Weltkrieg. Münster : Waxmann, 2015.

13. - BLIGAARD, Mette. Frederiksborgs genrejsning. Historicisme i teori og praksis, 1-2. Copenhague : Vandkunsten, 2008-2009.

14. - ARENDS, Isabel Maria. «Die Hase-Schule. Zur Reformgotik der hannoveranschen Architektenschule». Dans HÖHNS, Ulrich (éd.). Heinrich Moldenschardt: 1839-1891. Stilvolle Architektur in Schleswig-Holstein. Heide : Boyens, 2009.

15. - NITSCHE, Peter, KLUG, Ekkehard (éd.). Preussen in der Provinz. Beiträge zum 1. DeutschPolnischen Historikerkolloquium ... Francfort-sur-le-Main : Peter Lang, 1991.

16. - BARTH, Matthias. Kaiserliches Berlin. Architektur zwischen 1871 und 1918. Fribourg : Korn, 2012.

17. - BÖLKE, Stefan. Die Marineschule Mürwik. Architekturmonographische Untersuchung eines Repräsentationsbaues der Kaiserlichen Marine. Francfort-sur-le-Main : Peter Lang, 1998.

18. - MILLER LANE, Barbara. Op.cit., p. 204-206; ZIETZ, Peer. Franz Heinrich Schwechten. Ein Architekt zwischen Historismus und Moderne. Stuttgart: Axel Menges, 1999; ZERNACK, Juli. « Nordenschwärmerei und Germanenbegeisterung im Kaiserreich ». Dans HENNINGSEN, Bernd (éd.). Wahlverwandtschaft. Skandinavien und Deutschland 1800 bis 1914. Berlin : Jovis, 1997, p. 71-80 ; MAI, Ekkehard et WAETZOLDT, Stephan (éd.). Kunstverwaltung, Bau-und Denkmalpolitik im Kaiserreich. Berlin : Mann, 1981.

19. - WALLE, Heinrich. « Marinebauten in Kiel ». Dans ELVERT, Jürgen. Kiel, Die Deutschen und die See. Stuttgart: Franz Steiner, 1992, p. 207-234 ; DRAGSBO, Peter. « Tysk marinearkitektur 1904. Sønderborg Kasernes arkitektur mellem nationalromantik og moderne ». Dans PORSKROG RASMUSSEN, Carsten, et al. (éd.). Sønderborgs soldater. Kasernen i Sønderborg 1907-2014. Sønderborg : Museum Sønderjylland - Sønderborg Slot, 2015, p. 125-136.

20. - KAATMANN, Christian. Byggestil og byggeskik - nationale strømninger i sønderjysk arkitektur 1850-1940. Aabenraa : Institut for Grænseregionsforskning, 1988, p. 95.

21. - ANDRESEN, Hans Günther. Bauen in Backstein. Schleswig-Holsteinische Heimatschutz-Architektur zwischen Tradition und Reform. Heide : Boyens, 1989.

22. - Ibid., p. 48-52 et 70-85.

23. - DRAGSBO, Peter. En feelles kulturarv. Tyske og danske bygninger $i$ Sønderjylland 1864-1920. Sønderborg : Museum Sønderjylland - Sønderborg Slot, 2010/2011. 
24. - DRAGSBO, Peter. « Nieder mit Italien, Hoch Mögeltondern. Nordschleswig im Brennpunkt zwischen schleswig-holsteinischer Regionalarchitektur und dänischer Nationalarchitektur». Nordelbingen, 2009, p. 255-278.

25. - HÖHNS, Ulrich. « Lernen von Århus und Bernau ». Dans KÖSTER, Bernd. Op. cit., p. 102-136.

26. - SCHWENSEN, Broder et KÖSTER, Bernd (éd.). Paul Ziegler - Magistratsbaurat in Flensburg 1905-1939. Flensburg: Gesellschaft für Flensburger Stadtgeschichte, 1998, p. 18-79.

27. - PIGNON-FELLER, Christiane. Metz, 1900-1939. Un quartier impérial pour une nouvelle ville. Paris : Éditions du patrimoine, 2013.

28. - PIGNON-FELLER, Christiane. «Résister à la germanisation par l'architecture et les arts décoratifs ». Les Cahiers lorrains, 1-2, juin 2009, p. 38-49.

29. - NOHLEN, Klaus. Baupolitik im Reichsland Elsass-Lothringen 1870-1918. Die repräsentativeen Staatsbauten um den ehemaligen Kaiserplatz in Strassburg. Berlin : Mann, 1982 (publié en français en 1997) ; POTTECHER, Marie. "La Neustadt in Strasbourg ». Dans COHEN, Jean-Louis et FRANK, Hartmut. Op. cit., p. 174-181.

30. - Prenant son nom de l'ouvrage de MEBES, Paul. Um 1800. Architektur und Handwerk im letzten Jahrhundert ihrer traditionellen Entwicklung. Munich : F. Bruckmann, 1908.

31. - VOIGT, Wolfgang. Deutsche Architekten in Elsass 1940-1944. Planen und Bauen im annektierten Grenzland. Tübingen : Wasmuth, 2012.

32. - RENAN, Ernest. Qu'est-ce qu'une nation ? Conférence à la Sorbonne en 1882, postulant que la nationalité est un référendum quotidien, contre la position allemande qui prétendait que les populations d'Alsace-Lorraine étaient allemandes, en raison de leur langue et de leur culture et en dépit de leur propre conscience.

33. - BORN, Robert, LABUDA, Adam S. et STÖRTKUHL, Beate (éd.). Visuelle Erinnerungskulturen und Geschichtskonstruktionen in Deutschland und Polen 1800 bis 1939. Varsovie : Deutsches Kulturinstitut PAN, 2006; KEMLEIN, Sophia (éd.). Postkarten erzählen Geschichte. Die Stadt Posen 1896-1918. Lüneburg : Nordostdeutsches Kulturwerk, 1997.

34. - SKURATOWICZ, Jan. "Die wilhelminische Architektur in Polen». Dans NITSCHE, Peter, KLUG, Ekkehard (éd.). Preussen in der Provinz. Op. cit., p. 94-104.

35. - MOLIK, Witold. « Der Einfluss der preussischen Politik auf der Gesellschaftsstrukturen des Grossherzogtums Posen (1815-1914). Polnische Intelligenz als Vorbild ». Dans ibid., p. 63-79.

36. - CROWLEY, David. National Style and Nation-State. Design in Poland from the vernacular revival to the international style. Manchester/New York: Manchester University Press, 1992 ; MUTHESIUS, Stefan. «New international forms or traditional Polish styles?» Dans CAMPBELL, Louise (éd.). Twentieth-Century Architecture and its Histories. Otley, UK : Society of Architectural Historians of Great Britain, 2000, p. 223-250.

37. - EISTERER, Klaus (éd.). Tirol zwischen Diktatur und Demokratie (1930-1950). Innsbruck : Studien Verlag, 2002 ; MITTERER, Wittfrida (éd.). Megawatt und Widerstand, Die Ära der Gross-Kraftwerke in Südtirol. Bozen : Athesia, 2004.

38. - Le mot "déplaisant» utilisé ici est la traduction de "unbequem», dans HUSE, Norbert. Unbequeme Baudenkmale. Entsorgen? Pflegen? Schützen? Munich : C. H. Beck, 1997.

39. - Classé au titre des Monuments historiques en 1993.

40. - CASSAZ, Dominique et EBERHARDT, Sophie (éd.). Strasbourg. De la Grande-île à la Neustadt. Un patrimoine urbain exceptionnel. Lyon : Lieux Dits, 2013 ; LAUTON, Édith. Die Strassburger Neustadt. Einzelprojekte zum Kulturerbe vereint. Strasbourg: 2012; POTTECHER, Marie. «La Neustadt in Strasbourg ». Art. cit.

41. - MAZUR, Zbigniew (éd.). Das deutsche Kulturerbe in den polnischen West-und Nordgebieten. Wiesbaden : Harassowitz, 2003.

42. - DRAGSBO, Peter. En feelles kulturarv. Op. Cit., 2000/2001, p. 32-34. 
43. - VERDORFER, Martha. "Die Stadt als "öffentlicher Erinnerungsraum" am Beispiel der Landeshauptstadt Bozen». Dans EISTERER, Klaus (éd.). Tirol zwischen Diktatur und Demokratie (1930-1950). Op. cit., p. 187-200.

44. - Voir le site : http://www.siegesdenkmal.com/de.html [consulté le 04/02/2019].

45. - WILCKEN, Niels. Architektur im Grenzraum. Op. cit., p. 342-344.

46. - Interview avec Dr Tadeusz Zuchowski, de l'Institut des beaux-arts, université Adam Mickiewicz, Poznań, enregistrée le 21 octobre 2013.

\section{RÉSUMÉS}

L'analyse de l'architecture dans cinq régions frontalières d'Europe semble suggérer que les rapports entre nation et nationalité d'un côté, et architecture de l'autre, présentent des ressemblances et des différences très intéressantes. Ainsi, le Schleswig et la Lorraine, dans leurs divisions nationales, ont vu émerger un style architectural alternatif, en réaction aux politiques architecturales allemandes dominantes. À la différence du Schleswig, les architectes d'inspiration française ont choisi un style nostalgique "vieux Paris ", tandis que le mouvement danois, dans le Schleswig du Nord, est resté proche de l'architecture danoise de son époque. Toutefois, aucune des deux parties de la Lorraine n'a développé un style vernaculaire distinct. Le Schleswig et l'Alsace ont vu se développer en parallèle un style vernaculaire, accepté par tout le monde, même si le Heimatstil du Schleswig était toujours considéré de tendance allemande ou de tendance danoise par les deux groupes nationaux. En Alsace, le style vernaculaire exprimait un désir largement partagé d'autonomie. Si nous nous tournons vers Posen (Poznan) et la Prusse de l'Ouest et vers le Tyrol du Sud, nous constatons qu'aucun des mouvements irrédentistes de ces régions ne s'est préoccupé d'architecture. Leurs luttes s'attachaient à d'autres priorités : avant tout la langue, la propriété foncière et la présence publique. Les politiques architecturales allemandes, et surtout le romantisme national de l'Empereur Guillaume II et de son architecte Franz Schwechten, peuvent être comprises comme une tentative de résoudre le problème des différences historiques et culturelles au sein du Reich. Les manifestations du style wilhelmien furent particulièrement importantes dans les «forteresses frontalières » de Metz et de Posen. Niels Wilcken a interprété ce mécanisme comme l'expression d'une revendication de pouvoir douteuse dans des lieux où ce pouvoir était contesté. En dépit des frontières et des divisions nationales, toutefois, il ne faut pas négliger le puissant courant transversal des grands mouvements de l'architecture européenne, ni l'influence des académies d'architecture. Le régionalisme de l'Allemagne du sud a pu étendre son influence depuis l'académie de Munich en Bavière mais également en Autriche, dans le Tyrol et en Alsace. De même, le néo-classicisme vu à Strasbourg dans les années précédant la Première Guerre mondiale est un mouvement développé d'abord dans les académies de Stuttgart et de Karlsruhe et il est resté le style dominant sur les deux rives du Rhin jusqu'à la Deuxième Guerre. De manière similaire, le Heimatstil du Schleswig, fusionné avec le Bedre Byggeskik (style danois), fut employé partout jusqu'après la Deuxième Guerre. En regardant, enfin, cette architecture frontalière en tant que patrimoine, il faut se rappeler que chaque nation était responsable du patrimoine à l'intérieur de ses frontières, quelles que soient les origines nationales des créateurs des bâtiments et des monuments. En fait, le maintien d'un patrimoine "autre» devrait non seulement être accepté mais plus encore, vivement encouragé. La conscience de l'importance d'un patrimoine "étranger ", susceptible d'éclairer une histoire et une culture "indigène ", est capitale, et devrait faciliter une 
compréhension authentique de tous les partis. Les chercheurs, les services patrimoniaux et les historiens partagent une responsabilité dans la mission de faire des patrimoines nationaux un patrimoine commun.

Looking back at the five analyzed border regions, one might conclude that the relations between nation and nationality on one hand and architecture on the other hand demonstrate interesting similarities and differences. Thus, Schleswig and Lorraine had much in common regarding to national schism and the development of a counter-architectural style in opposition to the vociferous German architectural politics. Contrary to Schleswig, the French-minded architects chose a nostalgic 'old Paris' style, whereas the Danish movement in North Schleswig kept in close connection to contemporary Danish architecture. However, neither of the two parts in Lorraine developed a distinct vernacular style. Thus, Schleswig and Alsace underwent a more parallel development of a vernacular style, accepted by all, although the Schleswig Heimatstil was still considered either as a German-Schleswig style or a Danish-Schleswig style by the two national parts, whereas the Alsatian vernacular style rather symbolized a widespread wish for autonomy. Looking at Posen and Western Prussia as well as South Tyrol, none of the irredenta movements cared much about architecture, seeking the national battlefields in other arenas, in particular language, land-ownership and public presence. The German politics of architecture, especially the national romanticism of Emperor Wilhelm II and Franz Schwechten, can be seen as an attempt to solve the problem with the historical and cultural dissimilarity of the Reich. Nevertheless, the demonstrations of the imperial style were particularly directed towards the selected German 'border fortresses' of Metz and Poznan. Niels Wilcken has interpreted this mechanism as expression of a doubtfully legitimate claim for power in a border region with a disputed state of affairs'. In spite of all borders and national divides one should not forget, however, the strong transversal impact from the great currents of European architecture as well as the influence from the architectural academies. Thus, a South German regionalism extended its influence from the Munich academy not only to Bavaria, but to Austria, Tyrol and Alsace, too. Another example is the Strasbourg neo-classicism from the years before and after World War I, which was originally developed at the academies of Stuttgart and Karlsruhe and kept its status as the dominating style on both sides of the Rhine until World War II. Likewise the Schleswig Heimatstil - amalgamated with the Danish Bedre Byggeskik style - was usable for all national parts until the post-World War II years. Speaking lastly of border region architecture as heritage, one must underline that every nation has a responsibility for the heritage inside its borders, no matter which side created the actual buildings and monuments. In matter of fact, the maintenance of the heritage from the 'other parts' should not only be a accepted, but also a consciousness of the importance of the 'foreign' heritage that could highlight one's own history and culture is paramount, enabling a more genuine understanding of both sides. Therefore researchers, heritage authorities and historians have a common responsibility for turning national heritages into common heritage.

\section{INDEX}

Mots-clés : nation et architecture, architecture des frontières, architecture du pouvoir, contrearchitecture, architecture de contestation, régions germano-danoise du Schleswig, comparaison entre Schleswig/Alsace-Lorraine, Poznan et Tyrol du Sud, patrimoine encombrant (des frontières)

Keywords : nation and architecture, Architecture of border regions, Architecture of power / counter-architecture, (Danish-German region of) Schleswig, Comparison (between Schleswig, Alsace-Lorraine, Poznan and South Tyrolia), The difficult heritage (of borderlands) 


\section{AUTEUR}

\section{PETER DRAGSBO}

Conservateur honoraire du Musée Danois de la région frontière du Schleswig peter@dragsbo.dk 